\title{
A geometric aggregation operator for decision making
}

\author{
Ismat Beg $^{1}$ - Tabasam Rashid ${ }^{2}$
}

Received: 6 May 2015 / Accepted: 15 July 2015 / Published online: 1 August 2015

(C) The Author(s) 2015. This article is published with open access at Springerlink.com

\begin{abstract}
We proposed an aggregation operator which is used to aggregate decision makers' opinions in group decision making process. First, a Choquet integral-based distance between generalized interval-valued trapezoidal fuzzy numbers is defined. Then combining the generalized intervalvalued trapezoidal fuzzy number aggregation operator with Choquet integral-based distance, an extension of technique for order preference by similarity to ideal solution method is developed to deal with a multi-criteria generalized intervalvalued trapezoidal fuzzy number group decision making problems, where inter-dependent or interactive characteristics among criteria preference of decision makers are also considered. Finally, an illustrative example is provided to elaborate the proposed method.
\end{abstract}

Keywords Multi-criteria group decision making . Generalized interval-valued trapezoidal fuzzy numbers . Fuzzy measures - Technique for order preference by similarity to ideal solution

\section{Introduction}

Technique for order preference by similarity to ideal solution (TOPSIS) was initiated by Hwang and Yoon [21]. TOPSIS is a useful and practical technique for selection and ranking of alternatives [8]. The basic principle is that the chosen alterna-

$凶$ Ismat Beg

ibeg@lahoreschool.edu.pk; begismat@yahoo.com

Tabasam Rashid

tabasam.rashid@gmail.com

1 Lahore School of Economics, Lahore, Pakistan

2 University of Management and Technology, Lahore, Pakistan tive should have the shortest distance from the positive-ideal solution and the farthest distance from the negative-ideal solution. In the TOPSIS theory, crisp values are used for weights and performance ratings of the criteria. Hwang and Yoon [21] developed a classical approach to multiattribute/multi-criteria decision making (MADM/MCDM) problems by using TOPSIS. Often, human judgements and preferences are ambiguous, vague and cannot be estimated with exact numeric value under many conditions, so the crisp values are not suitable to model real-world situations. Zadeh [45] proposed the concept of fuzzy set theory and successfully used to handle imprecision (or uncertainty) in decision making problems, to solve the ambiguity and vagueness in information from human judgements and preferences. TOPSIS has been extended to fuzzy environment in [23]. Fuzzy numbers are applied and used to establish a prototype fuzzy TOPSIS [12,32]. Recently a lot of work on fuzzy TOPSIS has been developed by several authors $[6,16,30]$. Extension of TOPSIS method for triangular fuzzy numbers is developed in [10]. TOPSIS was further extended by Xu and Chen [42], an interactive method for fuzzy multiple attribute group decision making.

Interval-valued fuzzy TOPSIS method and experimental analysis has been proposed by Chen and Tsao [14] and they also gave the ranking technique of alternatives based on distance measure. Ashtiani et al. [2] gave an extension of fuzzy TOPSIS for interval-valued fuzzy sets. Chen and Lee [13] further developed interval type-2 TOPSIS method for fuzzy multiple attributes group decision making. On the basis of interval arithmetic, Chu and Lin [16] introduced the fuzzy TOPSIS model. The concept of interval-valued intuitionistic fuzzy sets (IVIFS) was given by Atanassov [4], as a generalization of IFS. The basic characteristic of the IVIFS is that the values of its membership function and nonmembership function are intervals rather than exact numbers. 
Some operational laws of the IVIFS are defined in [3]. In [38] a novel method for multiple attribute decision making based on IVIFS and TOPSIS method in uncertain environments is presented. Wei [41] applied IIFWGA aggregation functions to deal with dynamic multiple attribute decision making where all the attribute values are expressed in intuitionistic fuzzy numbers or interval-valued intuitionistic fuzzy numbers. TOPSIS-based nonlinear programming methodology for multi-attribute decision making with interval-valued intuitionistic fuzzy sets was proposed by Li [27]. Beg and Rashid [7] proposed the concept of trapezoidal valued intuitionistic fuzzy sets and used it for multi-criteria fuzzy decision making with Choquet integral-based TOPSIS. Recently, Li and Chen proposed a multi-criteria group decision making method based on trapezoidal intuitionistic fuzzy information in [28]

Several aggregation process are based on the assumption that the criteria (attribute) or preferences of decision makers are independent, and the aggregation operators are linear operators based on additive measures, which is characterized by an independence axiom [24,40]. For real decision making problems, there is a phenomenon that there exists some degree of inter-dependent or interactive characteristics between criteria [19]. Usually, decision makers invited from same or similar fields for a decision problem. They have similar social status, knowledge and preference. Their subjective preference show non-linearity. Independence phenomena among these criteria and mutual preferential independence of decision makers are thus violated. Sugeno [37] introduced the concept of non-additive fuzzy measure, which makes monotonicity instead of additivity property of fuzzy measure. It is a more effective tool to model interaction phenomena $[22,26]$ and to deal with decision making problems [19,33]. Liginlal and Ow [29] is an excellent review on analyzing decision maker behavior using fuzzy measure. In the real decision making problems, the attributes of the problem are often correlated or inter-dependent. Choquet integral [15] is a useful tool to model the correlation or inter-dependence. It has been studied and applied in the decision making methods $[9,17,31]$. Choquet integral is also used for interaction between criteria in hierarchy process [1]. Aggregation of decision makers' opinions is very important in group decision making problems to perform evaluation process. Group decision making involves weighted aggregation of all individual decisions to obtain a single collective decision. In [36], aggregation operator of intuitionistic fuzzy group decision making is proposed with the weights of decision makers. The weights of decision makers play an important role in the process of aggregation. In $[38,43]$, aggregation of the interval-valued intuitionistic fuzzy group decision making environment with the Choquet integral is studied.
The concept of interval-valued fuzzy number is extended to the concept of generalized fuzzy number in [44]. Furthermore, the concept of generalized fuzzy number is extended to the generalized interval-valued trapezoidal fuzzy numbers (GITFNs) by Chen [11]. GITFNs are suitable for imprecise and uncertain preference or information in multi-criteria decision method. Chen [11] extended TOPSIS method for multiple criteria decision analysis (MCDA) based on GITFNs. Recently, TOPSIS is modified for generalized interval-valued trapezoidal fuzzy numbers to select the most suitable robot in [34]. Dubois [18] gave some literature about some old and new techniques for fuzzy decision analysis. Moreover, Dubois discussed membership function, aggregation operators, linguistic variables, fuzzy intervals and the valued preference relations. By the motivation of new techniques of decision analysis in [18] and the use of Choquet integral in MCDM in $[38,39]$, we extended TOPSIS for GITFNs with aggregation operators and preference of decision makers. Combining this proposed aggregation operator with TOPSIS on Choquet integral-based distance, a multicriteria generalized interval-valued trapezoidal fuzzy group decision making is proposed, where interaction phenomena among the decision making problem and weights of decision makers are taken into account.

The rest of the article is organized as follows: in Sect. 2, we review fuzzy set, fuzzy measure, triangular norm, interval-valued fuzzy sets and generalized interval valued trapezoidal fuzzy numbers (GITFNs). In Sect. 3, we introduce an exponent law on GITFN, order relation and some of its properties are also studied in this section. In Sect. 4 , based on some operational laws, a generalized intervalvalued trapezoidal fuzzy number geometric aggregation (GITFNGA) operator is proposed, and some of its properties are examined. In Sect. 5, according to definition of Choquet integral, we define the Choquet integral-based distance between any two generalized interval-valued trapezoidal fuzzy sets. Combining the generalized interval-valued trapezoidal fuzzy geometric aggregation operator with Choquet integral-based distance, an extension of TOPSIS is developed to deal with a multi-criteria generalized interval-valued trapezoidal fuzzy group decision making problem where inter-dependent or interactive characteristics among criteria and preferences of decision makers are considered. In Sect. 6, an illustrative example is constructed to understand the application of the method and to demonstrate its practicality and feasibility.

\section{Preliminaries}

As preparation for introducing our new aggregation operators, some preliminary concepts are given in this section. 
Let $X$ be a crisp universe of generic elements, a fuzzy set $A$ in the universe $X$ is a mapping from $X$ to $[0,1]$. For any $x \in X$, the value $A(x)$ is called the degree of membership of $x$ in $A$.

Let $X=\left\{x_{1}, x_{2}, \ldots, x_{n}\right\}$ be the set of the attributes, $P(X)$ be the power set of $X$.

Definition 2.1 [37] A $\lambda$-fuzzy measure $\mu$ on the set $X$ is a set function $\mu: P(X) \rightarrow[0,1]$ satisfying the following properties:

1. $\mu(\phi)=0, \mu(X)=1$;

2. $B \subseteq C$ implies $\mu(B) \leq \mu(C)$, for all $B, C \subseteq X$;

3. $\mu(B \cup C)=\mu(B)+\mu(C)+\lambda \mu(B) \mu(C)$ for all $B, C \subseteq$ $X$ and $B \cap C=\phi$, where $\lambda \in(-1,+\infty)$.

In the above definition, if $\lambda=0$, then the third condition reduces to the axiom of the additive measure: $\mu(B \cup C)=$ $\mu(B)+\mu(C)$ for all $B, C \subseteq X$ and $B \cap C=\phi$, which indicates that there is no interaction between $B$ and $C$. Also $\lambda \neq 0$ indicates that the $\lambda$-fuzzy measure $\mu$ is non-additive and there is interaction between $B$ and $C$.

For $\lambda>0, \mu(B \cup C)>\mu(B)+\mu(C)$, which implies that $\mu$ is a super-additive measure. When $\lambda<0$, then $\mu(B \cup C)<$ $\mu(B)+\mu(C)$, which shows that $\mu$ is a sub-additive measure. The interaction between sets can be represented by $\lambda$.

If $X$ is a finite set, then $\bigcup_{i=1}^{n} x_{i}=X$. To determine $\lambda$-fuzzy measure $\mu$ on $X$, Sugeno [37] gave the following Eq. (2.1)

$$
\mu(X)=\mu\left(\bigcup_{i=1}^{n} x_{i}\right)= \begin{cases}\frac{1}{\lambda}\left\{\prod_{i=1}^{n}\left[1+\lambda \mu\left(x_{i}\right)\right]-1\right\} & \text { if } \lambda \neq 0, \\ \sum_{i=1}^{n} \mu\left(x_{i}\right) & \text { if } \lambda=0 .\end{cases}
$$

It can be noted that $\mu\left(x_{i}\right)$ for a subset with a single element $x_{i}$ is called a fuzzy density.

Specially for any subset $A \subset X$, we have

$$
\mu(A)= \begin{cases}\frac{1}{\lambda}\left\{\prod_{x_{i} \in A}\left[1+\lambda \mu\left(x_{i}\right)\right]-1\right\} & \text { if } \lambda \neq 0, \\ \sum_{x_{i} \in A} \mu\left(x_{i}\right) & \text { if } \lambda=0 .\end{cases}
$$

Based on Eq. (2.1), the value of $\lambda$ can be uniquely determined from $\mu(X)=1$, which is equivalent to solving

$$
1=\frac{1}{\lambda}\left\{\prod_{i=1}^{n}\left[1+\lambda \mu\left(x_{i}\right)\right]-1\right\} .
$$

If the elements of $B$ in $X$ are independent, we have

$\mu(B)=\sum_{x_{i} \in B} \mu\left(x_{i}\right)$, for all $B \subseteq X$

So the interaction between sets or elements of set depends upon the value of $\lambda$ and $\mu$.

Definition 2.2 [25] A binary operation $T:[0,1]^{2} \rightarrow[0,1]$ is a triangular norm ( $t$-norm) if it satisfies the following:

1. $T(1, x)=x$ for all $x \in[0,1]$ (boundary condition)

2. $T(x, y)=T(y, x)$ for all $x, y \in[0,1]$ (commutativity)

3. $T(x, T(y, z))=T(T(x, y), z)$ for all $x, y, z \in[0,1]$ (associativity)

4. if $w \leq x$ and $y \leq z$ then $T(w, y) \leq T(x, z)$ for all $w, x, y, z \in[0,1]$ (monotonicity).

Definition 2.3 [35] Let CS ([0,1]) stand for the set of all closed subintervals of $[0,1]$. Let $X=\left\{x_{1}, x_{2}, \ldots\right\}$ be a universe of discourse. An interval-valued fuzzy set (IVFS) in $X$ is an expression $A$ given by $A=\left\{\left\langle x, f_{A}(x)\right\rangle \mid x \in X\right\}$, where $f_{A}: X \rightarrow \operatorname{CS}([0,1])$. The numbers $f_{A}(x)$ represent the degree of membership of the element $x$ in the set $A$, such that $x \rightarrow f_{A}(x)=\left[f_{A}^{-}(x), f_{A}^{+}(x)\right]$. Set of all IVFSs on $X$ is denoted by $\operatorname{IVFS}(X)$.

Definition 2.4 [35] Let $A \in \operatorname{IVFS}(X)$. If $A(x)$ is a convex set and is defined in a closed and bounded interval, then $A$ is called "a generalized interval-valued fuzzy number (IVFN) on the universe of discourse $X$ ". Let $A(x)=$ $\left[A^{L}(x), A^{U}(x)\right]$, where $0 \leq A^{L}(x) \leq A^{U}(x) \leq 1, x \in X$, $A^{L}: X \rightarrow[0,1]$, and $A^{U}: X \rightarrow[0,1]$. All generalized IVFNs on $X$ are denoted by $\operatorname{IVFN}(X)$.

Definition 2.5 [11] Let $A^{L}\left(x_{i}\right)=\left(x_{1 i}^{L}, x_{2 i}^{L}, x_{3 i}^{L}, x_{4 i}^{L} ; h_{x_{i}}^{L}\right)$ and $A^{U}\left(x_{i}\right)=\left(x_{1 i}^{U}, x_{2 i}^{U}, x_{3 i}^{U}, x_{4 i}^{U} ; h_{x_{i}}^{U}\right)$ be two generalized trapezoidal fuzzy numbers. Let $h_{x_{i}}^{L}$ and $h_{x_{i}}^{U}$ denote the heights of $A^{L}\left(x_{i}\right)$ and $A^{U}\left(x_{i}\right)$, respectively. Let $x_{1 i}^{L}, x_{2 i}^{L}, x_{3 i}^{L}, x_{4 i}^{L}$, $x_{1 i}^{U}, x_{2 i}^{U}, x_{3 i}^{U}, x_{4 i}^{U} \in[0,1]$. A generalized interval-valued trapezoidal fuzzy set (GITFS) $A$ defined on the universe of discourse $X=\left\{x_{1}, x_{2}, \ldots\right\}$ is represented by the following:

$$
\begin{aligned}
A & =\left\{\left\langle x_{i},\left[A^{L}\left(x_{i}\right), A^{U}\left(x_{i}\right)\right]\right\rangle \mid x_{i} \in X\right\} \\
& =\left\{\left\langle x_{i},\left[\left(x_{1 i}^{L}, x_{2 i}^{L}, x_{3 i}^{L}, x_{4 i}^{L} ; h_{x_{i}}^{L}\right),\left(x_{1 i}^{U}, x_{2 i}^{U}, x_{3 i}^{U}, x_{4 i}^{U} ; h_{x_{i}}^{U}\right)\right]\right\rangle \mid x_{i} \in X\right\},
\end{aligned}
$$

where $x_{1 i}^{L} \leq x_{2 i}^{L} \leq x_{3 i}^{L} \leq x_{4 i}^{L}, x_{1 i}^{U} \leq x_{2 i}^{U} \leq x_{3 i}^{U} \leq x_{4 i}^{U}, 0 \leq h_{x_{i}}^{L}$ $\leq h_{x_{i}}^{U} \leq 1, x_{1 i}^{U} \leq x_{1 i}^{L}$, and $x_{4 i}^{L} \leq x_{4 i}^{U}$.

A GITFN $A(x)=\left[A^{L}(x), A^{U}(x)\right]$ consists of the two generalized trapezoidal fuzzy numbers $A^{L}(x)=\left(x_{1}^{L}, x_{2}^{L}\right.$, $\left.x_{3}^{L}, x_{4}^{L} ; h_{x}^{L}\right)$ and $A^{U}(x)=\left(x_{1}^{U}, x_{2}^{U}, x_{3}^{U}, x_{4}^{U} ; h_{x}^{U}\right)$, where 
$A^{L}(x)$ is called " the lower trapezoidal fuzzy number," and $A^{U}(x)$ is called " the upper trapezoidal fuzzy number".

If $h_{x}^{L}=h_{x}^{U}=1$, then the GITFN $A(x)$ becomes a normal interval-valued trapezoidal fuzzy number. If $A^{L}(x)=$ $A^{U}(x)$, then $A$ becomes a generalized trapezoidal fuzzy number. If $x_{2}^{L}=x_{3}^{L}$ and $x_{2}^{U}=x_{3}^{U}$, then $A(x)$ is a generalized interval-valued triangular fuzzy number. If $x_{2}^{L}=x_{3}^{L}$, $x_{2}^{U}=x_{3}^{U}$, and $h_{x}^{L}=h_{x}^{U}=1$, then $A$ is a normal intervalvalued triangular fuzzy number. If $x_{1}^{L}=x_{2}^{L}=x_{3}^{L}=x_{4}^{L}, x_{1}^{U}$ $=x_{2}^{U}=x_{3}^{U}=x_{4}^{U}$, and $h_{x}^{L}=h_{x}^{U}=1$, then $A^{L}(x)$ and $A^{U}(x)$ become crisp values, and $A(x)$ is a crisp interval.

We call $\left[\left(x_{1}^{L}, x_{2}^{L}, x_{3}^{L}, x_{4}^{L} ; h_{x}^{L}\right),\left(x_{1}^{U}, x_{2}^{U}, x_{3}^{U}, x_{4}^{U} ; h_{x}^{U}\right)\right]$ a GITFN. Let $\Omega$ be the set of all GITFNs. By Definition 2.5, $[(0,0,0,0 ; 0),(0,0,0,0 ; 0)]$ and $[(1,1,1,1 ; 1),(1,1,1$, $1 ; 1)]$ are the smallest and largest GITFNs, respectively.

Definition 2.6 [5] Let $\tilde{a}_{i}=\left[\left(a_{1 i}^{L}, a_{2 i}^{L}, a_{3 i}^{L}, a_{4 i}^{L} ; h_{a_{i}}^{L}\right),\left(a_{1 i}^{U}\right.\right.$, $\left.\left.a_{2 i}^{U}, a_{3 i}^{U}, a_{4 i}^{U} ; h_{a_{i}}^{U}\right)\right]$, and $\tilde{b}_{i}=\left[\left(b_{1 i}^{L}, b_{2 i}^{L}, b_{3 i}^{L}, b_{4 i}^{L} ; h_{b_{i}}^{L}\right),\left(b_{1 i}^{U}\right.\right.$, $\left.\left.b_{2 i}^{U}, b_{3 i}^{U}, b_{4 i}^{U} ; h_{b_{i}}^{U}\right)\right],(i=1,2, \ldots, n)$ be two GITFNs on $X$, then

$$
\begin{aligned}
d\left(\tilde{a}_{i}, \tilde{b}_{i}\right)=\frac{1}{8} & \left(\left|h_{a_{i}}^{L} \times a_{1 i}^{L}-b_{1 i}^{L} \times h_{b_{i}}^{L}\right|+\left|h_{a_{i}}^{L} \times a_{2 i}^{L}-b_{2 i}^{L} \times h_{b_{i}}^{L}\right|\right. \\
& +\left|h_{a_{i}}^{L} \times a_{3 i}^{L}-b_{3 i}^{L} \times h_{b_{i}}^{L}\right|+\left|h_{a_{i}}^{L} \times a_{4 i}^{L}-b_{4 i}^{L} \times h_{b_{i}}^{L}\right| \\
& +\left|h_{a_{i}}^{U} \times a_{1 i}^{U}-b_{1 i}^{U} \times h_{b_{i}}^{U}\right|+\left|h_{a_{i}}^{U} \times a_{2 i}^{U}-b_{2 i}^{U} \times h_{b_{i}}^{U}\right| \\
& \left.+\left|h_{a_{i}}^{U} \times a_{3 i}^{U}-b_{3 i}^{U} \times h_{b_{i}}^{U}\right|+\left|h_{a_{i}}^{U} \times a_{4 i}^{U}-b_{4 i}^{U} \times h_{b_{i}}^{U}\right|\right),
\end{aligned}
$$

is called the distance between $\tilde{a}_{i}$ and $\tilde{b}_{i}$.

\section{Properties of GITFNs}

Definition 3.1 For any two GITFNs, $\tilde{a}$ and $\tilde{b}$, where $\tilde{a}=$ $\left[\left(a_{1}^{L}, a_{2}^{L}, a_{3}^{L}, a_{4}^{L} ; h_{a}^{L}\right),\left(a_{1}^{U}, a_{2}^{U}, a_{3}^{U}, a_{4}^{U} ; h_{a}^{U}\right)\right]$, and $\tilde{b}=$ $\left[\left(b_{1}^{L}, b_{2}^{L}, b_{3}^{L}, b_{4}^{L} ; h_{b}^{L}\right),\left(b_{1}^{U}, b_{2}^{U}, b_{3}^{U}, b_{4}^{U} ; h_{b}^{U}\right)\right] ;$

1. If $a_{4}^{L} \leq b_{4}^{L}$ and $a_{4}^{U}<b_{4}^{U}$, then $\tilde{a}<\tilde{b}$;

2. If $a_{4}^{L} \leq b_{4}^{L}$ and $a_{4}^{U}>b_{4}^{U}$, then $\tilde{a}>\tilde{b}$;

3. If $a_{4}^{L}<b_{4}^{L}$ and $a_{4}^{U}=b_{4}^{U}$, then $\tilde{a}<\tilde{b}$;

4. If $a_{4}^{L}=b_{4}^{L}$ and $a_{4}^{U}=b_{4}^{U}$ and $h_{a}^{L}<h_{b}^{L}$, then $\tilde{a}<\tilde{b}$;

5. If $a_{4}^{L}=b_{4}^{L}$ and $a_{4}^{U}=b_{4}^{U}$ and $h_{a}^{L}=h_{b}^{L}$, then $\tilde{a}=\tilde{b}$.

Definition 3.2 Let $\tilde{a}=\left[\left(a_{1}^{L}, a_{2}^{L}, a_{3}^{L}, a_{4}^{L} ; h_{a}^{L}\right),\left(a_{1}^{U}, a_{2}^{U}, a_{3}^{U}\right.\right.$, $\left.\left.a_{4}^{U} ; h_{a}^{U}\right)\right]$, and $\tilde{b}=\left[\left(b_{1}^{L}, b_{2}^{L}, b_{3}^{L}, b_{4}^{L} ; h_{b}^{L}\right),\left(b_{1}^{U}, b_{2}^{U}, b_{3}^{U}, b_{4}^{U}\right.\right.$; $\left.h_{b}^{U}\right)$ ] be two GITFNs, then

1. $\tilde{a} \cdot \tilde{b}=\left[\left(a_{1}^{L} b_{1}^{L}, a_{2}^{L} b_{2}^{L}, a_{3}^{L} b_{3}^{L}, a_{4}^{L} b_{4}^{L} ; \min \left(h_{a}^{L}, h_{b}^{L}\right)\right)\right.$, $\left.\left(a_{1}^{U} b_{1}^{U}, a_{2}^{U} b_{2}^{U}, a_{3}^{U} b_{3}^{U}, a_{4}^{U} b_{4}^{U} ; \min \left(h_{a}^{U}, h_{b}^{U}\right)\right)\right]$;

2. $\tilde{a}^{\lambda}=\left[\left(\left(a_{1}^{L}\right)^{\lambda},\left(a_{2}^{L}\right)^{\lambda},\left(a_{3}^{L}\right)^{\lambda},\left(a_{4}^{L}\right)^{\lambda} ; h_{a}^{L}\right),\left(\left(a_{1}^{U}\right)^{\lambda},\left(a_{2}^{U}\right)^{\lambda}\right.\right.$, $\left.\left.\left(a_{3}^{U}\right)^{\lambda},\left(a_{4}^{U}\right)^{\lambda} ; h_{a}^{U}\right)\right]$, where $\lambda>0$.
Definition 3.2 is modified version [11, Definition 2.4.3].

For two operational laws of Definition 3.2, it is easy to obtain the following propositions.

Proposition 3.3 Let $\tilde{a}=\left[\left(a_{1}^{L}, a_{2}^{L}, a_{3}^{L}, a_{4}^{L} ; h_{a}^{L}\right),\left(a_{1}^{U}, a_{2}^{U}\right.\right.$, $\left.\left.a_{3}^{U}, a_{4}^{U} ; h_{a}^{U}\right)\right]$, and $\tilde{b}=\left[\left(b_{1}^{L}, b_{2}^{L}, b_{3}^{L}, b_{4}^{L} ; h_{b}^{L}\right),\left(b_{1}^{U}, b_{2}^{U}\right.\right.$, $\left.\left.b_{3}^{U}, b_{4}^{U} ; h_{b}^{U}\right)\right]$ be two GITFNs, and let $\tilde{c}=\tilde{a} \cdot \tilde{b}$ and $\tilde{d}=\tilde{a}^{\lambda}$, then both $\tilde{c}$ and $\tilde{d}$ are also GITFNs.

Proposition 3.4 Let $\tilde{a}=\left[\left(a_{1}^{L}, a_{2}^{L}, a_{3}^{L}, a_{4}^{L} ; h_{a}^{L}\right),\left(a_{1}^{U}, a_{2}^{U}\right.\right.$, $\left.\left.a_{3}^{U}, a_{4}^{U} ; h_{a}^{U}\right)\right]$, and $\tilde{b}=\left[\left(b_{1}^{L}, b_{2}^{L}, b_{3}^{L}, b_{4}^{L} ; h_{b}^{L}\right),\left(b_{1}^{U}, b_{2}^{U}, b_{3}^{U}\right.\right.$, $\left.\left.b_{4}^{U} ; h_{b}^{U}\right)\right]$ be two GITFNs. Then we have:

1. $\tilde{a} \cdot \tilde{b}=\tilde{b} \cdot \tilde{a}$

2. $(\tilde{a} \cdot \tilde{b})^{\lambda}=\tilde{a}^{\lambda} \cdot \tilde{b}^{\lambda}$

3. $\tilde{a}^{\lambda_{1}+\lambda_{2}}=\tilde{a}^{\lambda_{1}} \cdot \tilde{a}^{\lambda_{2}}$, for all $\lambda, \lambda_{1}, \lambda_{2}>0$.

\section{GITFNs geometric aggregation operator}

In the following, based on $\lambda$-fuzzy measure, we first give the definition of GITFNs geometric aggregation operator and then study its properties.

Definition 4.1 Let $\tilde{a}_{i}=\left[\left(a_{1 i}^{L}, a_{2 i}^{L}, a_{3 i}^{L}, a_{4 i}^{L} ; h_{a_{i}}^{L}\right),\left(a_{1 i}^{U}, a_{2 i}^{U}\right.\right.$, $\left.\left.a_{3 i}^{U}, a_{4 i}^{U} ; h_{a_{i}}^{U}\right)\right](i=1,2, \ldots, n)$ be a collection of GITFNs on $X$ and $\mu$ be a $\lambda$-fuzzy measure on $X$. Based on $\lambda$-fuzzy measure $\mu$, a GITFNs geometric aggregation (GITFNGA) operator of dimension $n$ is a mapping GITFNGA: $\Omega^{n} \rightarrow \Omega$ such that

$$
\begin{aligned}
& \operatorname{GITFNGA}_{\mu}\left(\tilde{a}_{1}, \tilde{a}_{2}, \ldots, \tilde{a}_{n}\right) \\
& =\left(\tilde{a}_{(1)}\right)^{\mu\left(A_{(1)}\right)-\mu\left(A_{(2)}\right)} \cdot\left(\tilde{a}_{(2)}\right)^{\mu\left(A_{(2)}\right)-\mu\left(A_{(3)}\right)} \\
& \quad \cdots\left(\tilde{a}_{(n)}\right)^{\mu\left(A_{(n)}\right)-\mu\left(A_{(n+1)}\right)}
\end{aligned}
$$

where ${ }_{(\cdot)}$ indicates a permutation on $X$ such that $\tilde{a}_{(1)} \leq \tilde{a}_{(2)} \leq$ $\cdots \leq \tilde{a}_{(n)}$ and $A_{(i)}=((i), \ldots,(n)), A_{(n+1)}=\phi$.

Theorem 4.2 Let $\tilde{a}_{i}=\left[\left(a_{1 i}^{L}, a_{2 i}^{L}, a_{3 i}^{L}, a_{4 i}^{L} ; h_{a_{i}}^{L}\right),\left(a_{1 i}^{U}, a_{2 i}^{U}\right.\right.$, $\left.\left.a_{3 i}^{U}, a_{4 i}^{U} ; h_{a_{i}}^{U}\right)\right](i=1,2, \ldots, n)$ be a collection of GITFNs on $X$, and $\mu$ be a $\lambda$-fuzzy measure on $X$, then their aggregated value by using the GITFNGA $A_{\mu}$ operator is also a GITFN, and

$$
\begin{gathered}
\operatorname{GITFNGA}_{\mu}\left(\tilde{a}_{1}, \tilde{a}_{2}, \ldots, \tilde{a}_{n}\right)=\left[\left(\prod_{i=1}^{n}\left(a_{1(i)}^{L}\right)^{\mu\left(A_{(i)}\right)-\mu\left(A_{(i+1)}\right)},\right.\right. \\
\prod_{i=1}^{n}\left(a_{2(i)}^{L}\right)^{\mu\left(A_{(i)}\right)-\mu\left(A_{(i+1)}\right)}, \prod_{i=1}^{n}\left(a_{3(i)}^{L}\right)^{\mu\left(A_{(i)}\right)-\mu\left(A_{(i+1)}\right)}, \\
\left.\prod_{i=1}^{n}\left(a_{4(i)}^{L}\right)^{\mu\left(A_{(i)}\right)-\mu\left(A_{(i+1)}\right)} ; \min _{i=1}^{n} h_{a_{i}}^{L}\right)
\end{gathered}
$$




$$
\begin{aligned}
& \left(\prod_{i=1}^{n}\left(a_{1(i)}^{U}\right)^{\mu\left(A_{(i)}\right)-\mu\left(A_{(i+1)}\right)}, \prod_{i=1}^{n}\left(a_{2(i)}^{U}\right)^{\mu\left(A_{(i)}\right)-\mu\left(A_{(i+1)}\right)},\right. \\
& \prod_{i=1}^{n}\left(a_{3(i)}^{U}\right)^{\mu\left(A_{(i)}\right)-\mu\left(A_{(i+1)}\right)} \\
& \left.\left.\prod_{i=1}^{n}\left(a_{4(i)}^{U}\right)^{\mu\left(A_{(i)}\right)-\mu\left(A_{(i+1)}\right)} ; \min _{i=1}^{n} h_{a_{i}}^{U}\right)\right]
\end{aligned}
$$

where (.) indicates a permutation on $X$ such that $\tilde{a}_{(1)} \leq \tilde{a}_{(2)}$ $\leq \cdots \leq \tilde{a}_{(n)}$ and $A_{(i)}=((i), \ldots,(n)), A_{(n+1)}=\phi$.

Proof The first result follows immediately from Definition 4.1 and Proposition 3.3. Next we prove Eq. (4.1) by using mathematical induction on $n$.

By the operational laws of Definition 3.2, we have

$$
\begin{aligned}
&\left(\tilde{a}_{(1)}\right)^{\mu\left(A_{(1)}\right)-\mu\left(A_{(2)}\right)} \\
&= {\left[\left(\left(a_{1(1)}^{L}\right)^{\mu\left(A_{(1)}\right)-\mu\left(A_{(2)}\right)},\left(a_{2(1)}^{L}\right)^{\mu\left(A_{(1)}\right)-\mu\left(A_{(2)}\right),}\right.\right.} \\
&\left.\left(a_{3(1)}^{L}\right)^{\mu\left(A_{(1)}\right)-\mu\left(A_{(2)}\right)},\left(a_{4(1)}^{L}\right)^{\mu\left(A_{(1)}\right)-\mu\left(A_{(2)}\right)} ; h_{a_{1}}^{L}\right), \\
&\left(\left(a_{1(1)}^{U}\right)^{\mu\left(A_{(1)}\right)-\mu\left(A_{(2)}\right)},\left(a_{2(1)}^{U}\right)^{\mu\left(A_{(1)}\right)-\mu\left(A_{(2)}\right)},\right. \\
&\left.\left.\left(a_{3(1)}^{U}\right)^{\mu\left(A_{(1)}\right)-\mu\left(A_{(2)}\right)},\left(a_{4(1)}^{U}\right)^{\mu\left(A_{(1)}\right)-\mu\left(A_{(2)}\right)} ; h_{a_{1}}^{U}\right)\right],
\end{aligned}
$$

$$
\begin{aligned}
\left(\tilde{a}_{(2)}\right)^{\mu\left(A_{(1)}\right)-\mu\left(A_{(2)}\right)} & {\left[\left(\left(a_{1(2)}^{L}\right)^{\mu\left(A_{(1)}\right)-\mu\left(A_{(2)}\right)},\left(a_{2(2)}^{L}\right)^{\mu\left(A_{(1)}\right)-\mu\left(A_{(2)}\right),}\right.\right.} \\
= & \left.\left(a_{3(2)}^{L}\right)^{\mu\left(A_{(1)}\right)-\mu\left(A_{(2)}\right)},\left(a_{4(2)}^{L}\right)^{\mu\left(A_{(1)}\right)-\mu\left(A_{(2)}\right)} ; h_{a_{2}}^{L}\right), \\
& \left(\left(a_{1(2)}^{U}\right)^{\mu\left(A_{(1)}\right)-\mu\left(A_{(2)}\right)},\left(a_{2(2)}^{U}\right)^{\mu\left(A_{(1)}\right)-\mu\left(A_{(2)}\right)},\right. \\
& \left.\left.\left(a_{3(2)}^{U}\right)^{\mu\left(A_{(1)}\right)-\mu\left(A_{(2)}\right)},\left(a_{4(2)}^{U}\right)^{\mu\left(A_{(1)}\right)-\mu\left(A_{(2)}\right)} ; h_{a_{2}}^{U}\right)\right] .
\end{aligned}
$$

Also

$$
\begin{aligned}
\tilde{a}_{1} \cdot \tilde{a}_{2}= & {\left[\left(a_{1(1)}^{L} a_{1(2)}^{L}, a_{2(1)}^{L} a_{2(2)}^{L}, a_{3(1)}^{L} a_{3(2)}^{L}, a_{4(1)}^{L} a_{4(2)}^{L} ;\right.\right.} \\
& \left.\min \left(h_{a_{1}}^{L}, h_{a_{2}}^{L}\right)\right),\left(a_{1(1)}^{U} a_{1(2)}^{U}, a_{2(1)}^{U} a_{2(2)}^{U},\right. \\
& \left.\left.a_{3(1)}^{U} a_{3(2)}^{U}, a_{4(1)}^{U} a_{4(2)}^{U} ; \min \left(h_{a_{1}}^{U}, h_{a_{2}}^{U}\right)\right)\right]
\end{aligned}
$$

For $n=2$ in Eq. (4.1), we have

$$
\begin{aligned}
& \operatorname{GITFNGA}_{\mu}\left(\tilde{a}_{1}, \tilde{a}_{2}\right)=\left(\tilde{a}_{1}\right)^{\mu\left(A_{(1)}\right)-\mu\left(A_{(2)}\right)} \cdot\left(\tilde{a}_{2}\right)^{\mu\left(A_{(2)}\right)-\mu\left(A_{(3)}\right)} \\
& =\left[\left(\left(a_{1(1)}^{L}\right)^{\mu\left(A_{(1)}\right)-\mu\left(A_{(2)}\right)} \cdot\left(a_{1(2)}^{L}\right)^{\mu\left(A_{(2)}\right)-\mu\left(A_{(3)}\right)}\right.\right.
\end{aligned}
$$

$$
\begin{aligned}
& \left(a_{2(1)}^{L}\right)^{\mu\left(A_{(1)}\right)-\mu\left(A_{(2)}\right)} \cdot\left(a_{2(2)}^{L}\right)^{\mu\left(A_{(2)}\right)-\mu\left(A_{(3)}\right)}, \\
& \left(a_{3(1)}^{L}\right)^{\mu\left(A_{(1)}\right)-\mu\left(A_{(2)}\right)} \cdot\left(a_{3(2)}^{L}\right)^{\mu\left(A_{(2)}\right)-\mu\left(A_{(3)}\right)}, \\
& \left.\left(a_{4(1)}^{L}\right)^{\mu\left(A_{(1)}\right)-\mu\left(A_{(2)}\right)} \cdot\left(a_{4(2)}^{L}\right)^{\mu\left(A_{(2)}\right)-\mu\left(A_{(3)}\right)} ; \min \left(h_{a_{1}}^{L}, h_{a_{2}}^{L}\right)\right), \\
& \left(\left(a_{1(1)}^{U}\right)^{\mu\left(A_{(1)}\right)-\mu\left(A_{(2)}\right)} \cdot\left(a_{1(2)}^{U}\right)^{\mu\left(A_{(2)}\right)-\mu\left(A_{(3)}\right)},\right. \\
& \left(a_{2(1)}^{U}\right)^{\mu\left(A_{(1)}\right)-\mu\left(A_{(2)}\right)} \cdot\left(a_{2(2)}^{U}\right)^{\mu\left(A_{(2)}\right)-\mu\left(A_{(3)}\right)}, \\
& \left(a_{3(1)}^{U}\right)^{\mu\left(A_{(1)}\right)-\mu\left(A_{(2)}\right)} \cdot\left(a_{3(2)}^{U}\right)^{\mu\left(A_{(2)}\right)-\mu\left(A_{(3)}\right)}, \\
& \left.\left.\left(a_{4(1)}^{U}\right)^{\mu\left(A_{(1)}\right)-\mu\left(A_{(2)}\right)} \cdot\left(a_{4(2)}^{U}\right)^{\mu\left(A_{(2)}\right)-\mu\left(A_{(3)}\right)} ; \min \left(h_{a_{1}}^{U}, h_{a_{2}}^{U}\right)\right)\right] .
\end{aligned}
$$

That is, for $n=2$, Eq. (4.1) holds.

Suppose that for $n=k$, Eq. (4.1) holds, i.e.,

$$
\begin{aligned}
\operatorname{GITFNGA}_{\mu}\left(\tilde{a}_{1}, \tilde{a}_{2}, \ldots, \tilde{a}_{k}\right) & {\left[\left(\prod_{i=1}^{k}\left(a_{1(i)}^{L}\right)^{\mu\left(A_{(i)}\right)-\mu\left(A_{(i+1)}\right)}, \prod_{i=1}^{k}\left(a_{2(i)}^{L}\right)^{\mu\left(A_{(i)}\right)-\mu\left(A_{(i+1)}\right)},\right.\right.} \\
& \left.\prod_{i=1}^{k}\left(a_{3(i)}^{L}\right)^{\mu\left(A_{(i)}\right)-\mu\left(A_{(i+1)}\right)}, \prod_{i=1}^{k}\left(a_{4(i)}^{L}\right)^{\mu\left(A_{(i)}\right)-\mu\left(A_{(i+1)}\right)} ; \min _{i=1}^{k} h_{a_{i}}^{L}\right), \\
& \left(\prod_{i=1}^{k}\left(a_{1(i)}^{U}\right)^{\mu\left(A_{(i)}\right)-\mu\left(A_{(i+1)}\right)}, \prod_{i=1}^{k}\left(a_{2(i)}^{U}\right)^{\mu\left(A_{(i)}\right)-\mu\left(A_{(i+1)}\right)},\right. \\
& \left.\left.\prod_{i=1}^{k}\left(a_{3(i)}^{U}\right)^{\mu\left(A_{(i)}\right)-\mu\left(A_{(i+1)}\right)}, \prod_{i=1}^{k}\left(a_{4(i)}^{U}\right)^{\mu\left(A_{(i)}\right)-\mu\left(A_{(i+1)}\right)} ; \min _{i=1}^{k} h_{a_{i}}^{U}\right)\right] .
\end{aligned}
$$

Then, for $n=k+1$, according to Definition 2.5, we have

$$
\begin{aligned}
& \operatorname{GITFNGA}_{\mu}\left(\tilde{a}_{1}, \tilde{a}_{2}, \ldots, \tilde{a}_{k+1}\right) \\
& =\left[\left(a_{1(k+1)}^{L}\right)^{\mu\left(A_{(k+1)}\right)-\mu\left(A_{(k+2)}\right)} \prod_{i=1}^{k}\left(a_{1(i)}^{L}\right)^{\mu\left(A_{(i)}\right)-\mu\left(A_{(i+1)}\right),}\right. \\
& \quad\left(a_{2(k+1)}^{L}\right)^{\mu\left(A_{(k+1)}\right)-\mu\left(A_{(k+2)}\right)} \prod_{i=1}^{k}\left(a_{2(i)}^{L}\right)^{\mu\left(A_{(i)}\right)-\mu\left(A_{(i+1)}\right)}, \\
& \quad\left(a_{3(k+1)}^{L}\right)^{\mu\left(A_{(k+1)}\right)-\mu\left(A_{(k+2)}\right)} \prod_{i=1}^{k}\left(a_{3(i)}^{L}\right)^{\mu\left(A_{(i)}\right)-\mu\left(A_{(i+1)}\right),} \\
& \quad\left(a_{4(k+1)}^{L}\right)^{\mu\left(A_{(k+1)}\right)-\mu\left(A_{(k+2)}\right)} \\
& \left.\prod_{i=1}^{k}\left(a_{4(i)}^{L}\right)^{\mu\left(A_{(i)}\right)-\mu\left(A_{(i+1)}\right)} ; \min \left(m_{i=1}^{k} h_{a_{i}}^{L}, h_{a_{k+1}}^{L}\right)\right) \\
& \quad\left(\left(a_{1(k+1)}^{U}\right)^{\mu\left(A_{(k+1)}\right)-\mu\left(A_{(k+2)}\right)} \prod_{i=1}^{k}\left(a_{1(i)}^{U}\right)^{\mu\left(A_{(i)}\right)-\mu\left(A_{(i+1)}\right)},\right. \\
& \quad\left(a_{2(k+1)}^{U}\right)^{\mu\left(A_{(k+1)}\right)-\mu\left(A_{(k+2)}\right)} \prod_{i=1}^{k}\left(a_{2(i)}^{U}\right)^{\mu\left(A_{(i)}\right)-\mu\left(A_{(i+1)}\right)}, \\
& \left(a_{3(k+1)}^{U}\right)^{\mu\left(A_{(k+1)}\right)-\mu\left(A_{(k+2))}\right)} \prod_{i=1}^{k}\left(a_{3(i)}^{U}\right)^{\mu\left(A_{(i)}\right)-\mu\left(A_{(i+1)}\right)},
\end{aligned}
$$




$$
\begin{aligned}
& \left(a_{4(k+1)}^{U}\right)^{\mu\left(A_{(k+1)}\right)-\mu\left(A_{(k+2)}\right)} \prod_{i=1}^{k}\left(a_{4(i)}^{U}\right)^{\mu\left(A_{(i)}\right)-\mu\left(A_{(i+1)}\right)} ; \\
& \left.\left.\min \left(\min _{i=1}^{k} h_{a_{i}}^{U}, h_{a_{k+1}}^{U}\right)\right]\right] \\
= & {\left[\prod_{i=1}^{k+1}\left(a_{1(i)}^{L}\right)^{\mu\left(A_{(i)}\right)-\mu\left(A_{(i+1)}\right)}, \prod_{i=1}^{k+1}\left(a_{2(i)}^{L}\right)^{\mu\left(A_{(i)}\right)-\mu\left(A_{(i+1)}\right),}\right.} \\
& \prod_{i=1}^{k+1}\left(a_{3(i)}^{L}\right)^{\mu\left(A_{(i)}\right)-\mu\left(A_{(i+1)}\right)}, \\
& \left.\prod_{i=1}^{k+1}\left(a_{4(i)}^{L}\right)^{\mu\left(A_{(i)}\right)-\mu\left(A_{(i+1)}\right)} ; \min _{i=1}^{k+1} h_{a_{i}}^{L}\right), \\
& \left(\prod_{i=1}^{k+1}\left(a_{1(i)}^{U}\right)^{\mu\left(A_{(i)}\right)-\mu\left(A_{(i+1)}\right)}, \prod_{i=1}^{k+1}\left(a_{2(i)}^{U}\right)^{\mu\left(A_{(i)}\right)-\mu\left(A_{(i+1)}\right)},\right. \\
& \prod_{i=1}^{k+1}\left(a_{3(i)}^{U}\right)^{\mu\left(A_{(i)}\right)-\mu\left(A_{(i+1)}\right)}, \\
& \left.\left.\prod_{i=1}^{k+1}\left(a_{4(i)}^{U}\right)^{\mu\left(A_{(i)}\right)-\mu\left(A_{(i+1)}\right)} ; \min _{i=1}^{k+1} h_{a_{i}}^{U}\right)\right] .
\end{aligned}
$$

That is, for $n=k+1$, Eq. (4.1) still holds.

Therefore, for all $n$, the Eq. (4.1) holds.

Remark 4.3 Let $\tilde{a}_{i}=\left[\left(a_{1 i}^{L}, a_{2 i}^{L}, a_{3 i}^{L}, a_{4 i}^{L} ; h_{a_{i}}^{L}\right),\left(a_{1 i}^{U}, a_{2 i}^{U}, a_{3 i}^{U}\right.\right.$, $\left.\left.a_{4 i}^{U} ; h_{a_{i}}^{U}\right)\right]$ and $\tilde{b}_{i}=\left[\left(b_{1 i}^{L}, b_{2 i}^{L}, b_{3 i}^{L}, b_{4 i}^{L} ; h_{b_{i}}^{L}\right),\left(b_{1 i}^{U}, b_{2 i}^{U}, b_{3 i}^{U}\right.\right.$, $\left.\left.b_{4 i}^{U} ; h_{b_{i}}^{U}\right)\right](i=1,2, \ldots, n)$ be two collections of GITFNs on $X . a_{j i}^{L}, a_{j i}^{U}, b_{j i}^{L}, b_{j i}^{U} \in[0,1]$ for any $i$ and $j=1,2,3,4$. If we assume that $T_{P}\left(a_{j i}^{U}, b_{j i}^{U}\right)=a_{j i}^{U} \cdot b_{j i}^{U}$, then $T_{P}$ is one of the basic $t$-norm, called the product, which satisfies the axioms of Definition 2.2. The associativity of $t$-norm allows us to extend the product $T_{P}$ in a unique way to an $n$-array operation in the usual way by induction. Define for each $n$-tuple $\left(x_{1}, x_{2}, \ldots, x_{n}\right) \in[0,1]^{n}$ :

$T_{P}\left(x_{1}, x_{2}, \ldots, x_{n}\right)=\prod_{i=1} x_{i}$.

Assume that $x_{1 i}^{U}=\left(a_{1(i)}^{U}\right)^{\mu\left(A_{(i)}\right)-\mu\left(A_{(i+1)}\right)}, \quad x_{2 i}^{U}=$ $\left(a_{2(i)}^{U}\right)^{\mu\left(A_{(i)}\right)-\mu\left(A_{(i+1)}\right)}, x_{3 i}^{U}=\left(a_{3(i)}^{U}\right)^{\mu\left(A_{(i)}\right)-\mu\left(A_{(i+1)}\right)}, x_{4 i}^{U}=$ $\left(a_{4(i)}^{U}\right)^{\mu\left(A_{(i)}\right)-\mu\left(A_{(i+1)}\right)}, x_{1 i}^{L}=\left(a_{1(i)}^{L}\right)^{\mu\left(A_{(i)}\right)-\mu\left(A_{(i+1)}\right)}, x_{2 i}^{L}=$ $\left(a_{2(i)}^{L}\right)^{\mu\left(A_{(i)}\right)-\mu\left(A_{(i+1)}\right)}, x_{3 i}^{L}=\left(a_{3(i)}^{L}\right)^{\mu\left(A_{(i)}\right)-\mu\left(A_{(i+1)}\right)}, x_{4 i}^{L}=$ $\left(a_{4(i)}^{L}\right)^{\mu\left(A_{(i)}\right)-\mu\left(A_{(i+1)}\right)}$. Now Theorem 4.2 further implies

$$
\begin{aligned}
\operatorname{GITFNGA}_{\mu}\left(\tilde{a}_{1}, \tilde{a}_{2}, \ldots, \tilde{a}_{n}\right) \\
=\left[\left(T_{P}\left(x_{11}^{L}, x_{12}^{L}, \ldots, x_{1 n}^{L}\right), T_{P}\left(x_{21}^{L}, x_{22}^{L}, \ldots, x_{2 n}^{L}\right),\right.\right. \\
\left.\quad T_{P}\left(x_{31}^{L}, x_{32}^{L}, \ldots, x_{3 n}^{L}\right), T_{P}\left(x_{41}^{L}, x_{42}^{L}, \ldots, x_{4 n}^{L}\right) ; \min _{i=1}^{n} h_{a_{i}}^{L}\right),
\end{aligned}
$$

$$
\begin{aligned}
& \left(T_{P}\left(x_{11}^{U}, x_{12}^{U}, \ldots, x_{1 n}^{U}\right), T_{P}\left(x_{21}^{U}, x_{22}^{U}, \ldots, x_{2 n}^{U}\right),\right. \\
& \left.\left.T_{P}\left(x_{31}^{U}, x_{32}^{U}, \ldots, x_{3 n}^{U}\right), T_{P}\left(x_{41}^{U}, x_{42}^{U}, \ldots, x_{4 n}^{U}\right) ; \min _{i=1}^{n} h_{a_{i}}^{U}\right)\right] .
\end{aligned}
$$

Thus the GITFNGA $\mu$ operator can be represented by one of the basic $t$-norm $T_{P}$.

Proofs of the following corollaries can be done easily by using the results from Sect. 3, Definition 4.1 and Theorem 4.2.

Corollary 4.4 Let $\tilde{a}_{i}=\left[\left(a_{1 i}^{L}, a_{2 i}^{L}, a_{3 i}^{L}, a_{4 i}^{L} ; h_{a_{i}}^{L}\right),\left(a_{1 i}^{U}, a_{2 i}^{U}\right.\right.$, $\left.\left.a_{3 i}^{U}, a_{4 i}^{U} ; h_{a_{i}}^{U}\right)\right](i=1,2, \ldots, n)$ be a collection of $G I T F N$ on $X$. If all $\tilde{a}_{i}$ are equal $(i=1,2, \ldots, n)$ such that $h_{a_{i}}^{L}=$ $h_{a_{k}}^{L}$ and $h_{a_{i}}^{U}=h_{a_{k}}^{U}$ and $a_{j i}^{L}=a_{j k}^{L}$ and $a_{j i}^{U}=a_{j k}^{U}$ for all $i=1$, $2, \ldots, n, k=1,2, \ldots, n$ and $j=1,2,3,4$, that $i s, \tilde{a}_{(i)}=$ $\tilde{a}_{(k)}$. Thus, for all $i, \tilde{a}_{i}=\tilde{a}=\left[\left(a_{1}^{L}, a_{2}^{L}, a_{3}^{L}, a_{4}^{L} ; h_{a}^{L}\right),\left(a_{1}^{U}\right.\right.$, $\left.\left.a_{2}^{U}, a_{3}^{U}, a_{4}^{U} ; h_{a}^{U}\right)\right]$. Therefore

$\operatorname{GITFNGA}_{\mu}\left(\tilde{a}_{1}, \tilde{a}_{2}, \ldots, \tilde{a}_{n}\right)=\tilde{a}$.

Corollary 4.5 Let $\tilde{a}_{i}=\left[\left(a_{1 i}^{L}, a_{2 i}^{L}, a_{3 i}^{L}, a_{4 i}^{L} ; h_{a_{i}}^{L}\right),\left(a_{1 i}^{U}, a_{2 i}^{U}\right.\right.$, $\left.\left.a_{3 i}^{U}, a_{4 i}^{U} ; h_{a_{i}}^{U}\right)\right]$ and $\tilde{b}_{i}=\left[\left(b_{1 i}^{L}, b_{2 i}^{L}, b_{3 i}^{L}, b_{4 i}^{L} ; h_{b_{i}}^{L}\right),\left(b_{1 i}^{U}, b_{2 i}^{U}\right.\right.$, $\left.\left.b_{3 i}^{U}, b_{4 i}^{U} ; h_{b_{i}}^{U}\right)\right](i=1,2, \ldots, n)$ be two collections of $G$ IT $F N$ on $X$, and $\mu$ be a $\lambda$-fuzzy measure on $X$. Let (.) indicate a permutation on $X$ such that $\tilde{a}_{(1)} \leq \cdots \leq \tilde{a}_{(n)}$ and $\tilde{b}_{(1)} \leq \cdots$ $\leq \tilde{b}_{(n)}$. If $a_{j i}^{L} \leq b_{j i}^{L}$ and $a_{j i}^{U} \leq b_{j i}^{U}$ for all $i$ and $j=1,2,3$, 4 , that is, $\tilde{a}_{(i)} \leq \tilde{b}_{(i)}$, then

$\operatorname{GITFNGA}_{\mu}\left(\tilde{a}_{1}, \tilde{a}_{2}, \ldots, \tilde{a}_{n}\right) \leq \operatorname{GITFNGA}_{\mu}\left(\tilde{b}_{1}, \tilde{b}_{2}, \ldots, \tilde{b}_{n}\right)$.

Corollary 4.6 Let $\tilde{a}_{i}=\left[\left(a_{1 i}^{L}, a_{2 i}^{L}, a_{3 i}^{L}, a_{4 i}^{L} ; h_{a_{i}}^{L}\right),\left(a_{1 i}^{U}, a_{2 i}^{U}\right.\right.$, $\left.\left.a_{3 i}^{U}, a_{4 i}^{U} ; h_{a_{i}}^{U}\right)\right](i=1,2, \ldots, n)$ be a collection of $G I T F N$ on $X$ and $\mu$ be a $\lambda$-fuzzy measure on $X$. If

$$
\begin{aligned}
\tilde{a}^{-}= & {\left[\left(\min _{i} a_{1 i}^{L}, \min _{i} a_{2 i}^{L}, \min _{i} a_{3 i}^{L}, \min _{i} a_{4 i}^{L} ; \min _{i} h_{a_{i}}^{L}\right),\right.} \\
& \left.\left(\min _{i} a_{1 i}^{U}, \min _{i} a_{2 i}^{U}, \min _{i} a_{3 i}^{U}, \min _{i} a_{4 i}^{U} ; \min _{i} h_{a_{i}}^{U}\right)\right] \\
\tilde{a}^{+}= & {\left[\left(\max _{i} a_{1 i}^{L}, \max _{i} a_{2 i}^{L}, \max _{i} a_{3 i}^{L}, \max _{i} a_{4 i}^{L} ; \max _{i} h_{a_{i}}^{L}\right),\right.} \\
& \left.\left(\max _{i} a_{1 i}^{U}, \max _{i} a_{2 i}^{U}, \max _{i} a_{3 i}^{U}, \max _{i} a_{4 i}^{U} ; \max _{i} h_{a_{i}}^{U}\right)\right]
\end{aligned}
$$

then

$\tilde{a}^{-} \leq \operatorname{GITFNGA}_{\mu}\left(\tilde{a}_{1}, \tilde{a}_{2}, \ldots, \tilde{a}_{n}\right) \leq \tilde{a}^{+}$

Corollary 4.7 Let $\tilde{a}_{i}=\left[\left(a_{1 i}^{L}, a_{2 i}^{L}, a_{3 i}^{L}, a_{4 i}^{L} ; h_{a_{i}}^{L}\right),\left(a_{1 i}^{U}, a_{2 i}^{U}\right.\right.$, $\left.\left.a_{3 i}^{U}, a_{4 i}^{U} ; h_{a_{i}}^{U}\right)\right](i=1,2, \ldots, n)$ be a collection of $G I T F N s$ 
on $X$ and $\mu$ be a $\lambda$-fuzzy measure on $X$. If $\tilde{s}=\left[\left(a_{1}^{L}, a_{2}^{L}, a_{3}^{L}\right.\right.$, $\left.\left.a_{4}^{L} ; h_{s}^{L}\right),\left(a_{1}^{U}, a_{2}^{U}, a_{3}^{U}, a_{4}^{U} ; h_{s}^{U}\right)\right]$ is a GIT FN on X, then

$$
\begin{gathered}
\operatorname{GTIFGA}_{\mu}\left(\tilde{a}_{1} \cdot \tilde{s}, \tilde{a}_{2} \cdot \tilde{s}, \ldots, \tilde{a}_{n} \cdot \tilde{s}\right) \\
=\operatorname{GTIFGA}_{\mu}\left(\tilde{a}_{1}, \tilde{a}_{2}, \ldots, \tilde{a}_{n}\right) \cdot \tilde{s} .
\end{gathered}
$$

Corollary 4.8 Let $\tilde{a}_{i}=\left[\left(a_{1 i}^{L}, a_{2 i}^{L}, a_{3 i}^{L}, a_{4 i}^{L} ; h_{a_{i}}^{L}\right),\left(a_{1 i}^{U}, a_{2 i}^{U}\right.\right.$, $\left.\left.a_{3 i}^{U}, a_{4 i}^{U} ; h_{a_{i}}^{U}\right)\right](i=1,2, \ldots, n)$ be a collection of $G I T F N s$ on $X$ and $\mu$ be a $\lambda$-fuzzy measure on $X$. If $v>0$, then

$$
\begin{aligned}
& \operatorname{GITFNGA}_{\mu}\left(\left(\tilde{a}_{1}\right)^{v},\left(\tilde{a}_{2}\right)^{v}, \ldots,\left(\tilde{a}_{n}\right)^{v}\right) \\
& =\left(\operatorname{GITFNGA}_{\mu}\left(\tilde{a}_{1}, \tilde{a}_{2}, \ldots, \tilde{a}_{n}\right)\right)^{v} .
\end{aligned}
$$

Corollary 4.9 Let $\tilde{a}_{i}=\left[\left(a_{1 i}^{L}, a_{2 i}^{L}, a_{3 i}^{L}, a_{4 i}^{L} ; h_{a_{i}}^{L}\right),\left(a_{1 i}^{U}, a_{2 i}^{U}\right.\right.$, $\left.\left.a_{3 i}^{U}, a_{4 i}^{U} ; h_{a_{i}}^{U}\right)\right](i=1,2, \ldots, n)$ be a collection of $G I T F N s$ on $X$ and $\mu$ be a $\lambda$-fuzzy measure on $X$. If $v>0$ and $\tilde{s}=$ $\left[\left(a_{1}^{L}, a_{2}^{L}, a_{3}^{L}, a_{4}^{L} ; h_{s}^{L}\right),\left(a_{1}^{U}, a_{2}^{U}, a_{3}^{U}, a_{4}^{U} ; h_{s}^{U}\right)\right]$ is a GITFN on $X$, then

$$
\begin{aligned}
& \operatorname{GITFNGA}_{\mu}\left(\left(\tilde{a}_{1}\right)^{v} \cdot \tilde{s},\left(\tilde{a}_{2}\right)^{v} \cdot \tilde{s}, \ldots,\left(\tilde{a}_{n}\right)^{v} \cdot \tilde{s}\right) \\
& \quad=\left(\operatorname{GITFNGA}_{\mu}\left(\tilde{a}_{1}, \tilde{a}_{2}, \ldots, \tilde{a}_{n}\right)\right)^{v} \cdot \tilde{s} .
\end{aligned}
$$

Corollary 4.10 Let $\tilde{a}_{i}=\left[\left(a_{1 i}^{L}, a_{2 i}^{L}, a_{3 i}^{L}, a_{4 i}^{L} ; h_{a_{i}}^{L}\right),\left(a_{1 i}^{U}, a_{2 i}^{U}\right.\right.$, $\left.\left.a_{3 i}^{U}, a_{4 i}^{U} ; h_{a_{i}}^{U}\right)\right](i=1,2, \ldots, n)$ be a collection of $G I T F N s$ on $X$ and $\mu$ be a $\lambda$-fuzzy measure on $X$.

1. If $\mu(A)=1$ for any $A \in P(X)$, then

$$
\begin{aligned}
& \operatorname{GITFNGA}_{\mu}\left(\tilde{a}_{1}, \tilde{a}_{2}, \ldots, \tilde{a}_{n}\right)=\left[\left(a_{1(n)}^{L}, a_{2(n)}^{L}, a_{3(n)}^{L},\right.\right. \\
& \left.\left.\times a_{4(n)}^{L} ; \min _{i=1}^{n} h_{a_{i}}^{L}\right),\left(a_{1(n)}^{U}, a_{2(n)}^{U}, a_{3(n)}^{U}, a_{4(n)}^{U} ; \min _{i=1}^{n} h_{a_{i}}^{U}\right)\right]
\end{aligned}
$$

2. If $\mu(A)=0$ for any $A \in P(X)$ and $A \neq X$, then

$$
\begin{aligned}
& \operatorname{GITFNGA}_{\mu}\left(\tilde{a}_{1}, \tilde{a}_{2}, \ldots, \tilde{a}_{n}\right)=\left[\left(a_{1(1)}^{L}, a_{2(1)}^{L}, a_{3(1)}^{L},\right.\right. \\
& \left.\left.\times a_{4(1)}^{L} ; \min _{i=1}^{n} h_{a_{i}}^{L}\right),\left(a_{1(1)}^{U}, a_{2(1)}^{U}, a_{3(1)}^{U}, a_{4(1)}^{U} ; \min _{i=1}^{n} h_{a_{i}}^{U}\right)\right]
\end{aligned}
$$

3. For any $A, B \in P(X)$ such that $|A|=|B|$, if $\mu(A)=$ $\mu(B)$ and $\mu\{(i), \ldots,(n)\}=\frac{n-i+1}{n}, 1 \leq i \leq n$, then

$$
\begin{aligned}
& \operatorname{GITFNGA}_{\mu}\left(\tilde{a}_{1}, \tilde{a}_{2}, \ldots, \tilde{a}_{n}\right) \\
&=\left[\left(\prod_{i=1}^{n}\left(a_{1(i)}^{L}\right)^{\frac{1}{n}}, \prod_{i=1}^{n}\left(a_{2(i)}^{L}\right)^{\frac{1}{n}}, \prod_{i=1}^{n}\left(a_{3(i)}^{L}\right)^{\frac{1}{n}}, \prod_{i=1}^{n}\left(a_{4(i)}^{L}\right)^{\frac{1}{n}} ; \min _{i=1}^{n} h_{a_{i}}^{L}\right),\right. \\
&\left.\left(\prod_{i=1}^{n}\left(a_{1(i)}^{U}\right)^{\frac{1}{n}}, \prod_{i=1}^{n}\left(a_{2(i)}^{U}\right)^{\frac{1}{n}}, \prod_{i=1}^{n}\left(a_{3(i)}^{U}\right)^{\frac{1}{n}}, \prod_{i=1}^{n}\left(a_{4(i)}^{U}\right)^{\frac{1}{n}} ; \min _{i=1}^{n} h_{a_{i}}^{U}\right)\right]
\end{aligned}
$$

\section{GITFN group decision making process}

It is reasonable to employ the Choquet integral in terms of the $\lambda$-fuzzy measure to aggregate the performance values instead of the weighted average method, since the Choquet integral does not assume the independence of one element from another.Choquet integral is defined as follows.

Definition 5.1 [19] Let $X=\left\{x_{1}, x_{2}, \ldots, x_{n}\right\}$ be a universe of discourse, $g$ be a positive real-valued function on $X$, and $\mu$ be a $\lambda$-fuzzy measure on $X$. The discrete Choquet integral of $g$ with respect to $\mu$ is defined by

$C_{\mu}(g)=\sum_{i=1}^{n} g\left(x_{(i)}\right)\left[\mu\left(A_{(i)}\right)-\mu\left(A_{(i+1)}\right)\right]$,

where (.) indicates a permutation on $X$ such that $g\left(x_{(1)}\right) \leq$ $g\left(x_{(2)}\right) \leq \cdots \leq g\left(x_{(n)}\right)$. Also $A_{(i)}=\left\{x_{(i)}, \ldots, x_{(n)}\right\}, A_{(n+1)}$ $=\phi$.

For further properties of Chouquet integral we refer to [7,9,19,31].

In general, multi-criteria group decision making problem includes uncertain and imprecise data and information. We consider the multi-criteria group decision making problems where all the criteria values are expressed in GITFNs, and interaction phenomena among the decision making criteria or preference of decision makers are taken into account. The following notations are used to depict the considered problems:

$E=\left\{e_{1}, e_{2}, \ldots, e_{r}\right\}$ is the set of the experts involved in the decision process;

$A=\left\{a_{1}, a_{2}, \ldots, a_{m}\right\}$ is the set of the considered alternatives;

$C=\left\{c_{1}, c_{2}, \ldots, c_{n}\right\}$ is the set of the criteria used for evaluating the alternatives.

In group decision making problems, aggregation of expert opinions is very important to appropriately perform evaluation process. In the following, Choquet integral-based TOPSIS is proposed for multi-criteria GIT $F N$ group decision making where expert opinions are aggregated by the GIT F NGA operator, which involves the following steps:

Step 1. As for every alternative $a_{i}(i=1,2, \ldots, m)$, each expert $e_{k}(k=1,2, \ldots, r)$, is invited to express his/her individual evaluation or preference according to each criteria $c_{j}(j=1,2, \ldots, n)$, by a $G I T F N s$ $\tilde{a}_{i j}^{k}=\left[\left(a_{1 i j^{k}}^{L}, a_{2 i j^{k}}^{L}, a_{3 i j^{k}}^{L}, a_{4 i j^{k}}^{L} ; h_{a_{i j k}}^{L}\right),\left(a_{1 i j^{k}}^{U}, a_{2 i j^{k}}^{U}\right.\right.$, $\left.\left.a_{3 i j^{k}}^{U}, a_{4 i j^{k}}^{U} ; h_{a_{i j k}}^{U}\right)\right](i=1,2, \ldots, m ; j=1,2, \ldots$, $n ; k=1,2, \ldots, r)$, where $\left[\left(a_{1 i j^{k}}^{L}, a_{2 i j^{k}}^{L}, a_{3 i j^{k}}^{L}, a_{4 i j^{k}}^{L}\right.\right.$; $\left.\left.h_{a_{i j k}}^{L}\right),\left(a_{1 i j^{k}}^{U}, a_{2 i j^{k}}^{U}, a_{3 i j^{k}}^{U}, a_{4 i j^{k}}^{U} ; h_{a_{i j k}}^{U}\right)\right]$ indicates the uncertain degree that expert $e_{k}$ considers what the alternative $a_{i}$ should satisfy the criteria $c_{j}$. Then we 
can obtain a decision making matrix as follows:

$$
R^{k}=\left(\begin{array}{cccc}
\tilde{a}_{11}^{k} & \tilde{a}_{12}^{k} & \cdots & \tilde{a}_{1 n}^{k} \\
\tilde{a}_{21}^{k} & \tilde{a}_{22}^{k} & \cdots & \tilde{a}_{2 n}^{k} \\
\vdots & \vdots & \ddots & \vdots \\
\tilde{a}_{m 1}^{k} & \tilde{a}_{m 2}^{k} & \cdots & \tilde{a}_{m n}^{k}
\end{array}\right)
$$

Step 2. Confirm the fuzzy density $\mu_{k}=\mu\left(e_{k}\right)$ of each expert. According to Eq. (2.3), parameter $\lambda_{1}$ of expert can be determined.

Step 3. By Definition 3.1, $\tilde{a}_{i j}^{k}$ is reordered such that $\tilde{a}_{i j}^{(k)} \leq$ $\tilde{a}_{i j}^{(k+1)}$.

Utilize the generalized interval-valued trapezoidal fuzzy Choquet integral operator

$$
\begin{aligned}
& \tilde{a}_{i j}=\operatorname{GITFNGA}_{\mu}\left(\tilde{a}_{i j}^{1}, \tilde{a}_{i j}^{2}, \ldots, \tilde{a}_{i j}^{r}\right) \\
& =\left[\left(\prod_{k=1}^{r}\left(a_{1 i j}^{L}\right)^{\mu\left(A_{(k)}\right)-\mu\left(A_{(k+1)}\right)},\right.\right. \\
& \prod_{k=1}^{r}\left(a_{2 i j(k)}^{L}\right)^{\mu\left(A_{(k)}\right)-\mu\left(A_{(k+1)}\right)}, \\
& \prod_{k=1}^{r}\left(a_{3 i j(k)}^{L}\right){ }^{\mu\left(A_{(k)}\right)-\mu\left(A_{(k+1)}\right)}, \\
& \left.\prod_{i=1}^{n}\left(a_{4 i j^{(k)}}^{L}\right)^{\mu\left(A_{(k)}\right)-\mu\left(A_{(k+1)}\right)} ; \min _{i=1}^{n} h_{a_{i j}(k)}^{L}\right) \text {, } \\
& \left(\prod_{i=1}^{n}\left(a_{1 i j(k)}^{U}\right)^{\mu\left(A_{(k)}\right)-\mu\left(A_{(k+1)}\right)},\right. \\
& \prod_{i=1}^{n}\left(a_{2 i j}^{U}\right)^{\mu\left(A_{(k)}\right)-\mu\left(A_{(k+1)}\right)}, \\
& \prod_{i=1}^{n}\left(a_{3 i j^{(k)}}^{U}\right)^{\mu\left(A_{(k)}\right)-\mu\left(A_{(k+1)}\right)}, \\
& \left.\left.\prod_{i=1}^{n}\left(a_{4 i j(k)}^{U}\right)^{\mu\left(A_{(k)}\right)-\mu\left(A_{(k+1)}\right)} ; \min _{i=1}^{n} h_{a_{i j}(k)}^{U}\right)\right]
\end{aligned}
$$

to aggregate all the GITFN decision matrices $R^{k}$ $=\left(\tilde{a}_{i j}^{k}\right)_{m \times n}(k=1,2, \ldots, r)$ into a complex GITFN decision matrix $R^{k}=\left(\tilde{a}_{i j}^{k}\right)_{m \times n}$, where $\tilde{a}_{i j}=$ $\left[\left(a_{1 i j}^{L}, a_{2 i j}^{L}, a_{3 i j}^{L}, a_{4 i j}^{L} ; h_{a_{i j}}^{L}\right),\left(a_{1 i j}^{U}, a_{2 i j}^{U}, a_{3 i j}^{U}, a_{4 i j}^{U}\right.\right.$; $\left.\left.h_{a_{i j}}^{U}\right)\right](i=1,2, \ldots, m ; j=1,2, \ldots, n), A_{(k)}=$ $\left\{e_{(k)}, \ldots, e_{(r)}\right\}, A_{(r+1)}=\phi$, and $\mu\left(A_{(k)}\right)$ can be calculated by Eq. (2.2).

Step 4. Let $J_{1}$ be a collection of benefit criteria (i.e., the larger $c_{j}$, the greater preference) and $J_{2}$ be a collection of cost criteria (i.e., the smaller $c_{j}$, the greater preference). The generalized interval-valued trapezoidal fuzzy positive-ideal solution (GIT-FPIS), denoted as $\tilde{\alpha}^{+}=\left(\tilde{\alpha}_{1}^{+} \tilde{\alpha}_{2}^{+} \ldots \tilde{\alpha}_{n}^{+}\right)$, and the generalized interval-valued trapezoidal fuzzy negative-ideal solution (GIT-FNIS), denoted as $\tilde{\alpha}^{-}=\left(\tilde{\alpha}_{1}^{-} \tilde{\alpha}_{2}^{-}\right.$ $\left.\ldots \tilde{\alpha}_{n}^{-}\right)$, are defined as follows:

$$
\begin{aligned}
\tilde{\alpha}^{+}= & {\left[\left(\left(\max _{i} a_{1 i j}^{L}, \max _{i} a_{2 i j}^{L}, \max _{i} a_{3 i j}^{L}, \max _{i} a_{4 i j}^{L} ; \max _{i} h_{a_{i j}}^{L}\right) \mid j \in J_{1},\right.\right.} \\
& \left.\left(\min _{i} a_{1 i j}^{L}, \min _{i} a_{2 i j}^{L}, \min _{i} a_{3 i j}^{L}, \min _{i} a_{4 i j}^{L} ; \min _{i} h_{a_{i j}}^{L}\right) \mid j \in J_{2}\right), \\
& \left(\left(\max _{i} a_{1 i j}^{U}, \max _{i} a_{2 i j}^{U}, \max _{i} a_{3 i j}^{U}, \max _{i} a_{4 i j}^{U} ; \max _{i} h_{a_{i j}}^{U}\right) \mid j \in J_{1},\right. \\
& \left.\left.\left(\min _{i} a_{1 i j}^{U}, \min _{i} a_{2 i j}^{U}, \min _{i} a_{3 i j}^{U}, \min _{i} a_{4 i j}^{U} ; \min _{i} h_{a_{i j}}^{U}\right) \mid j \in J_{2}\right)\right] \\
& i=1,2, \ldots, m, \\
\tilde{\alpha}^{+}= & \left(\tilde{\alpha}_{1}^{+} \tilde{\alpha}_{2}^{+} \ldots \tilde{\alpha}_{n}^{+}\right),
\end{aligned}
$$

where $\tilde{\alpha}_{j}^{+}=\left[\left(\alpha_{1 j}^{L+}, \alpha_{2 j}^{L+}, \alpha_{3 j}^{L+}, \alpha_{4 j}^{L+} ; h_{\alpha_{j}}^{L+}\right),\left(\alpha_{1 j}^{U+}\right.\right.$, $\left.\left.\alpha_{2 j}^{U+}, \alpha_{3 j}^{U+}, \alpha_{4 j}^{U+} ; h_{\alpha_{j}}^{U+}\right)\right](j=1,2, \ldots, n)$. $\tilde{\alpha}^{-}=\left[\left(\left(\min _{i} a_{1 i j}^{L}, \min _{i} a_{2 i j}^{L}, \min _{i} a_{3 i j}^{L}, \min _{i} a_{4 i j}^{L} ; \min _{i} h_{a_{i j}}^{L}\right) \mid j \in J_{1}\right.\right.$, $\left.\left(\max _{i} a_{1 i j}^{L}, \max _{i} a_{2 i j}^{L}, \max _{i} a_{3 i j}^{L}, \max _{i} a_{4 i j}^{L} ; \max _{i} h_{a_{i j}}^{L}\right) \mid j \in J_{2}\right)$, $\left(\left(\min _{i} a_{1 i j}^{U}, \min _{i} a_{2 i j}^{U}, \min _{i} a_{3 i j}^{U}, \min _{i} a_{4 i j}^{U} ; \min _{i} h_{a_{i j}}^{U}\right) \mid j \in J_{1}\right.$, $\left.\left.\left(\max _{i} a_{1 i j}^{U}, \max _{i} a_{2 i j}^{U}, \max _{i} a_{3 i j}^{U}, \max _{i} a_{4 i j}^{U} ; \max _{i} h_{a_{i j}}^{U}\right) \mid j \in J_{2}\right)\right]$ $i=1,2, \ldots, m$,

$\tilde{\alpha}^{-}=\left(\begin{array}{llll}\tilde{\alpha}_{1}^{-} & \tilde{\alpha}_{2}^{-} & \ldots & \tilde{\alpha}_{n}^{-}\end{array}\right)$

where $\tilde{\alpha}_{j}^{-}=\left[\left(\alpha_{1 j}^{L-}, \alpha_{2 j}^{L-}, \alpha_{3 j}^{L-}, \alpha_{4 j}^{L-} ; h_{\alpha_{j}}^{L-}\right),\left(\alpha_{1 j}^{U-}\right.\right.$, $\left.\left.\alpha_{2 j}^{U-}, \alpha_{3 j}^{U-}, \alpha_{4 j}^{U-} ; h_{\alpha_{j}}^{U-}\right)\right](j=1,2, \ldots, n)$.

Moreover, we denote the alternatives $a_{i}(i=1,2$, $\ldots, m)$ by $x_{i}=\left(\tilde{a}_{i 1}, \tilde{a}_{i 2}, \ldots, \tilde{a}_{i n}\right)$.

Step 5. Confirm the fuzzy density $\mu_{j}=\mu\left(c_{j}\right)$ of each criterion. According to Eq. (2.3), parameter $\lambda_{2}$ of criteria can be determined.

Step 6. Calculate the distance between the alternative $x_{i}$ and the GIT-FPIS $\tilde{\alpha}^{+}$and the distance between the alternative $x_{i}$ and the GIT-FNIS $\tilde{\alpha}^{-}$, respectively:

$d_{i}\left(x_{i}, \tilde{\alpha}^{+}\right)=\sum_{j=1}^{n} d_{i(j)}\left(\tilde{a}_{i j}, \tilde{\alpha}_{j}^{+}\right)\left(\mu\left(A_{(j)}\right)-\mu\left(A_{(j+1)}\right)\right)$,

where $d_{i j}\left(\tilde{a}_{i j}, \tilde{\alpha}_{j}^{+}\right)=\frac{1}{8}\left(\left|\alpha_{1 j}^{L+} \times h_{\alpha_{j}}^{L+}-a_{1 i j}^{L} \times h_{a_{i j}}^{L}\right|\right.$ $+\left|\alpha_{2 j}^{L+} \times h_{\alpha_{j}}^{L+}-a_{2 i j}^{L} \times h_{a_{i j}}^{L}\right|+\mid \alpha_{3 j}^{L+} \times h_{\alpha_{j}}^{L+}-$ $a_{3 i j}^{L} \times h_{a_{i j}}^{L}|+| \alpha_{4 j}^{L+} \times h_{\alpha_{j}}^{L+}-a_{4 i j}^{L} \times h_{a_{i j}}^{L}|+| \alpha_{1 j}^{U+} \times$ $h_{\alpha_{j}}^{U+}-a_{1 i j}^{U} \times h_{a_{i j}}^{U}|+| \alpha_{2 j}^{U+} \times h_{\alpha_{j}}^{U+}-a_{2 i j}^{U} \times h_{a_{i j}}^{U} \mid$ $+\left|\alpha_{3 j}^{U+} \times h_{\alpha_{j}}^{U+}-a_{3 i j}^{U} \times h_{a_{i j}}^{U}\right|+\mid \alpha_{4 j}^{U+} \times h_{\alpha_{j}}^{U+}-$ $\left.a_{4 i j}^{U} \times h_{a_{i j}}^{U} \mid\right)$, so that $d_{i(1)}\left(\tilde{a}_{i j}, \tilde{\alpha}_{j}^{+}\right) \leq d_{i(2)}\left(\tilde{a}_{i j}, \tilde{\alpha}_{j}^{+}\right) \leq$ 
$\cdots \leq d_{i(n)}\left(\tilde{a}_{i j}, \tilde{\alpha}_{j}^{+}\right), A_{(j)}=\left\{c_{(j)}, \ldots, c_{(n)}\right\}, A_{(n+1)}$

$=\phi, \mu\left(A_{(j)}\right)$ can be calculated by Eq. (2.2)

$$
\begin{aligned}
d_{i}\left(x_{i}, \tilde{\alpha}^{-}\right)= & \sum_{j=1}^{n} d_{i(j)}\left(\tilde{a}_{i j}, \tilde{\alpha}_{j}^{-}\right)\left(\mu\left(A_{(j)}\right)\right. \\
& \left.-\mu\left(A_{(j+1)}\right)\right)
\end{aligned}
$$

where $d_{i j}\left(\tilde{a}_{i j}, \tilde{\alpha}_{j}^{-}\right)=\frac{1}{8}\left(\left|\alpha_{1 j}^{L-} \times h_{\alpha_{j}}^{L-}-a_{1 i j}^{L} \times h_{a_{i j}}^{L}\right|\right.$ $+\left|\alpha_{2 j}^{L-} \times h_{\alpha_{j}}^{L-}-a_{2 i j}^{L} \times h_{a_{i j}}^{L}\right|+\mid \alpha_{3 j}^{L-} \times h_{\alpha_{j}}^{L-}-$ $a_{3 i j}^{L} \times h_{a_{i j}}^{L}|+| \alpha_{4 j}^{L-} \times h_{\alpha_{j}}^{L-}-a_{4 i j}^{L} \times h_{a_{i j}}^{L}|+| \alpha_{1 j}^{U-} \times$ $h_{\alpha_{j}}^{U-}-a_{1 i j}^{U} \times h_{a_{i j}}^{U}|+| \alpha_{2 j}^{U-} \times h_{\alpha_{j}}^{U-}-a_{2 i j}^{U} \times h_{a_{i j}}^{U} \mid+$ $\left|\alpha_{3 j}^{U-} \times h_{\alpha_{j}}^{U-}-a_{3 i j}^{U} \times h_{a_{i j}}^{U}\right|+\mid \alpha_{4 j}^{U-} \times h_{\alpha_{j}}^{U-}-a_{4 i j}^{U} \times$ $\left.h_{a_{i j}}^{U} \mid\right)$, so that $d_{i(1)}\left(\tilde{a}_{i j}, \tilde{\alpha}_{j}^{-}\right) \leq d_{i(2)}\left(\tilde{a}_{i j}, \tilde{\alpha}_{j}^{-}\right) \leq \cdots \leq$ $d_{i(n)}\left(\tilde{a}_{i j}, \tilde{\alpha}_{j}^{-}\right), A_{(j)}=\left\{c_{(j)}, \ldots, c_{(n)}\right\}, A_{(n+1)}=\phi$, $\mu\left(A_{(j)}\right)$ can be calculated by Eq. (2.2)

Step 7. Calculate the closeness coefficient of each alternative:

$$
r\left(x_{i}\right)=\frac{d_{i}\left(x_{i}, \tilde{\alpha}^{-}\right)}{d_{i}\left(x_{i}, \tilde{\alpha}^{+}\right)+d_{i}\left(x_{i}, \tilde{\alpha}^{-}\right)}, \quad i=1,2, \ldots, m .
$$

Step 8. Rank all the alternatives $a_{i}(i=1,2, \ldots, m)$ according to the closeness coefficient $r\left(x_{i}\right)$, the greater the value $r\left(x_{i}\right)$, the better the alternative $a_{i}$.

\section{Illustrative example}

An investment company, wants to invest money in the best option (adapted from [20]). There is possibility to invest the money in five sectors: firstly in car industry, secondly in food company, thirdly in computer company, fourthly in arms company, and lastly in TV company. The decision of investment company will be based on: (1) the risk analysis; (2) the growth analysis; (3) the social-political impact analysis, (4) the environmental impact analysis. There are three Board members who will decide where to invest. Now we utilized the proposed method where inter-dependent or interactive characteristics among criteria and preference of decision makers are taken into account to get the most desirable alternative.

Step 1. As there are five possible alternatives in which to invest the money: $a_{1}$ is a car industry, $a_{2}$ is a food company, $a_{3}$ is a computer company, $a_{4}$ is an arms company, $a_{5}$ is a TV company. The decision will be according to the following four criteria: $c_{1}$ is the risk analysis; $c_{2}$ is the growth analysis; $c_{3}$ is the socialpolitical impact analysis; $c_{4}$ is the environmental
Table 1 The decision matrix $R^{(1)}$

\begin{tabular}{lllll}
\hline & $c_{1}$ & $c_{2}$ & $c_{3}$ & $c_{4}$ \\
\hline$a_{1}$ & $\mathrm{M}$ & $\mathrm{G}$ & $\mathrm{P}$ & $\mathrm{P}$ \\
$a_{2}$ & $\mathrm{P}$ & $\mathrm{M}$ & $\mathrm{M}$ & $\mathrm{G}$ \\
$a_{3}$ & $\mathrm{G}$ & $\mathrm{M}$ & $\mathrm{VG}$ & $\mathrm{P}$ \\
$a_{4}$ & $\mathrm{VG}$ & $\mathrm{P}$ & $\mathrm{P}$ & $\mathrm{M}$ \\
$a_{5}$ & $\mathrm{EG}$ & $\mathrm{P}$ & $\mathrm{VP}$ & $\mathrm{G}$ \\
\hline
\end{tabular}

Table 2 The decision matrix $R^{(2)}$

\begin{tabular}{lllll}
\hline & $c_{1}$ & $c_{2}$ & $c_{3}$ & $c_{4}$ \\
\hline$a_{1}$ & $\mathrm{P}$ & $\mathrm{VG}$ & $\mathrm{VP}$ & $\mathrm{M}$ \\
$a_{2}$ & $\mathrm{VP}$ & $\mathrm{P}$ & $\mathrm{G}$ & $\mathrm{VG}$ \\
$a_{3}$ & $\mathrm{M}$ & $\mathrm{P}$ & $\mathrm{G}$ & $\mathrm{VP}$ \\
$a_{4}$ & $\mathrm{EG}$ & $\mathrm{M}$ & $\mathrm{P}$ & $\mathrm{G}$ \\
$a_{5}$ & $\mathrm{G}$ & $\mathrm{M}$ & $\mathrm{P}$ & $\mathrm{VG}$ \\
\hline
\end{tabular}

Table 3 The decision matrix $R^{(3)}$

\begin{tabular}{lllll}
\hline & $c_{1}$ & $c_{2}$ & $c_{3}$ & $c_{4}$ \\
\hline$a_{1}$ & $\mathrm{G}$ & $\mathrm{VG}$ & $\mathrm{M}$ & $\mathrm{VP}$ \\
$a_{2}$ & $\mathrm{M}$ & $\mathrm{P}$ & $\mathrm{VG}$ & $\mathrm{M}$ \\
$a_{3}$ & $\mathrm{P}$ & $\mathrm{VG}$ & $\mathrm{G}$ & $\mathrm{VP}$ \\
$a_{4}$ & $\mathrm{G}$ & $\mathrm{G}$ & $\mathrm{P}$ & $\mathrm{M}$ \\
$a_{5}$ & $\mathrm{M}$ & $\mathrm{P}$ & $\mathrm{M}$ & $\mathrm{EG}$ \\
\hline
\end{tabular}

impact analysis. The five possible alternatives $a_{i}(i$ $=1,2,3,4,5)$ are to be evaluated using the linguistic information by three decision makers (Board members) $e_{k}(k=1,2,3)$, as listed in Tables 1,2 and 3 .

$R^{1}, R^{2}$ and $R^{3}$ are matrices which are constructed by using Tables 1, 2, 3 and 4 .

$R^{1}=\left(\begin{array}{l}{[(0.4025,0.4525,0.5375,0.5675 ; 0.8),(0.32,0.41,0.58,0.65 ; 1.0)]} \\ {[(0.0875,0.1200,0.1600,0.1825 ; 0.8),(0.04,0.10,0.18,0.23 ; 1.0)]} \\ {[(0.7825,0.8150,0.8850,0.9075 ; 0.8),(0.72,0.78,0.92,0.97 ; 1.0)]} \\ {[(0.9475,0.9850,0.9925,0.9925 ; 0.8),(0.930,0.980,1.0,1.0 ; 1.0)]} \\ {[(1.0000,1.0000,1.0000,1.0000 ; 1.0),(1.000,1.000,1.0,1.0 ; 1.0)]}\end{array}\right.$

$[(0.7825,0.8150,0.8850,0.9075 ; 0.8),(0.72,0.78,0.92,0.97 ; 1.0)]$ $[(0.4025,0.4525,0.5375,0.5675 ; 0.8),(0.32,0.41,0.58,0.65 ; 1.0)]$ $[(0.4025,0.4525,0.5375,0.5675 ; 0.8),(0.32,0.41,0.58,0.65 ; 1.0)]$ $[(0.0875,0.1200,0.1600,0.1825 ; 0.8),(0.04,0.10,0.18,0.23 ; 1.0)]$ $[(0.0875,0.1200,0.1600,0.1825 ; 0.8),(0.04,0.10,0.18,0.23 ; 1.0)]$

$[(0.0875,0.1200,0.1600,0.1825 ; 0.8),(0.04,0.10,0.18,0.23 ; 1.0)]$ $[(0.4025,0.4525,0.5375,0.5675 ; 0.8),(0.32,0.41,0.58,0.65 ; 1.0)]$ $[(0.9475,0.9850,0.9925,0.9925 ; 0.8),(0.930,0.980,1.0,1.0 ; 1.0)]$ $[(0.0875,0.1200,0.1600,0.1825 ; 0.8),(0.04,0.10,0.18,0.23 ; 1.0)]$ $[(0.0075,0.0075,0.015,0.0525 ; 0.8),(0.000,0.00,0.02,0.07 ; 1.0)]$

$[(0.0875,0.1200,0.1600,0.1825 ; 0.8),(0.04,0.10,0.18,0.23 ; 1.0)]$ $[(0.7825,0.8150,0.8850,0.9075 ; 0.8),(0.72,0.78,0.92,0.97 ; 1.0)]$ $[(0.0875,0.1200,0.1600,0.1825 ; 0.8),(0.04,0.10,0.18,0.23 ; 1.0)]$ $[(0.4025,0.4525,0.5375,0.5675 ; 0.8),(0.32,0.41,0.58,0.65 ; 1.0)]$ $[(0.7825,0.8150,0.8850,0.9075 ; 0.8),(0.72,0.78,0.92,0.97 ; 1.0)])$ 
Table 4 The relationship between GITFNs and degrees of linguistic importance in a nine linguistic term scale is from Chen [11]

\begin{tabular}{ll}
\hline Linguistic terms & Generalized interval-valued trapezoidal fuzzy numbers \\
\hline Absolutely poor (AP) & {$[(0.0,0.0,0.0,0.0 ; 1.0),(0.0,0.0,0.0,0.0 ; 1.0)]$} \\
Very poor (VP) & {$[(0.0075,0.0075,0.015,0.0525 ; 0.8),(0.0,0.0,0.02,0.07 ; 1.0)]$} \\
Poor (P) & {$[(0.0875,0.12,0.16,0.1825 ; 0.8),(0.04,0.10,0.18,0.23 ; 1.0)]$} \\
Medium poor (MP) & {$[(0.2325,0.255,0.325,0.3575 ; 0.8),(0.17,0.22,0.36,0.42 ; 1.0)]$} \\
Medium (M) & {$[(0.4025,0.4525,0.5375,0.5675 ; 0.8),(0.32,0.41,0.58,0.65 ; 1.0)]$} \\
Medium good (MG) & {$[(0.65,0.6725,0.7575,0.79 ; 0.8),(0.58,0.63,0.80,0.86 ; 1.0)]$} \\
Good (G) & {$[(0.7825,0.815,0.885,0.9075 ; 0.8),(0.72,0.78,0.92,0.97 ; 1.0)]$} \\
Very good (VG) & {$[(0.9475,0.985,0.9925,0.9925 ; 0.8),(0.93,0.98,1.0,1.0 ; 1.0)]$} \\
Absolutely good (AG) & {$[(1.0,1.0,1.0,1.0 ; 1.0),(1.0,1.0,1.0,1.0 ; 1.0)]$} \\
\hline
\end{tabular}

$R^{2}=\left(\begin{array}{l}{[(0.0875,0.1200,0.1600,0.1825 ; 0.8),(0.04,0.10,0.18,0.23 ; 1.0)]} \\ {[(0.0075,0.0075,0.0150,0.0525 ; 0.8),(0.0,0.0,0.020,0.070 ; 1.0)]} \\ {[(0.4025,0.4525,0.5375,0.5675 ; 0.8),(0.32,0.41,0.58,0.65 ; 1.0)]} \\ {[(1.0000,1.0000,1.0000,1.0000 ; 1.0),(1.00,1.00,1.00,1.00 ; 1.0)]} \\ {[(0.7825,0.8150,0.8850,0.9075 ; 0.8),(0.72,0.78,0.92,0.97 ; 1.0)]}\end{array}\right.$

$[(0.9475,0.9850,0.9925,0.9925 ; 0.8),(0.93,0.98,1.00,1.00 ; 1.0)]$ $[(0.0875,0.1200,0.1600,0.1825 ; 0.8),(0.04,0.10,0.18,0.23 ; 1.0)]$ $[(0.0875,0.1200,0.1600,0.1825 ; 0.8),(0.04,0.10,0.18,0.23 ; 1.0)]$ $[(0.4025,0.4525,0.5375,0.5675 ; 0.8),(0.32,0.41,0.58,0.65 ; 1.0)]$ $[(0.4025,0.4525,0.5375,0.5675 ; 0.8),(0.32,0.41,0.58,0.65 ; 1.0)]$

$[(0.0075,0.0075,0.015,0.0525 ; 0.8),(0.0,0.0,0.020,0.07 ; 1.0)]$ $[(0.7825,0.815,0.885,0.9075 ; 0.8),(0.72,0.78,0.92,0.97 ; 1.0)]$ $[(0.7825,0.815,0.885,0.9075 ; 0.8),(0.72,0.78,0.92,0.97 ; 1.0)]$ $[(0.0875,0.1200,0.16,0.1825 ; 0.8),(0.04,0.10,0.18,0.23 ; 1.0)]$ $[(0.0875,0.120,0.160,0.1825 ; 0.8),(0.04,0.10,0.18,0.23 ; 1.0)]$

$[(0.4025,0.4525,0.5375,0.5675 ; 0.8),(0.32,0.41,0.58,0.65 ; 1.0)]$ $[(0.9475,0.9850,0.9925,0.9925 ; 0.8),(0.930,0.980,1.0,1.0 ; 1.0)]$ $[(0.0075,0.0075,0.0150,0.0525 ; 0.8),(0.0,0.0,0.020,0.070 ; 1.0)]$ $[(0.7825,0.8150,0.8850,0.9075 ; 0.8),(0.72,0.78,0.92,0.97 ; 1.0)]$ $[(0.9475,0.9850,0.9925,0.9925 ; 0.8),(0.930,0.980,1.0,1.0 ; 1.0)]$

$R^{3}=\left(\begin{array}{l}{[(0.7825,0.8150,0.8850,0.9075 ; 0.8),(0.72,0.78,0.92,0.97 ; 1.0)]} \\ {[(0.4025,0.4525,0.5375,0.5675 ; 0.8),(0.32,0.41,0.58,0.65 ; 1.0)]} \\ {[(0.0875,0.1200,0.1600,0.1825 ; 0.8),(0.04,0.10,0.18,0.23 ; 1.0)]} \\ {[(0.7825,0.8150,0.8850,0.9075 ; 0.8),(0.72,0.78,0.92,0.97 ; 1.0)]} \\ {[(0.4025,0.4525,0.5375,0.5675 ; 0.8),(0.32,0.41,0.58,0.65 ; 1.0)]}\end{array}\right.$

$[(0.9475,0.985,0.9925,0.9925 ; 0.8),(0.93,0.980,1.0,1.0 ; 1.0)]$ $[(0.0875,0.120,0.1600,0.1825 ; 0.8),(0.04,0.1,0.18,0.23 ; 1.0)]$ $[(0.9475,0.985,0.9925,0.9925 ; 0.8),(0.93,0.980,1.0,1.0 ; 1.0)]$ $[(0.7825,0.815,0.885,0.9075 ; 0.8),(0.72,0.78,0.92,0.97 ; 1.0)]$ $[(0.0875,0.120,0.160,0.1825 ; 0.8),(0.04,0.10,0.18,0.23 ; 1.0)]$

$[(0.4025,0.4525,0.5375,0.5675 ; 0.8),(0.32,0.41,0.58,0.65 ; 1.0)]$ $[(0.9475,0.9850,0.9925,0.9925 ; 0.8),(0.930,0.980,1.0,1.0 ; 1.0)]$ $[(0.7825,0.8150,0.8850,0.9075 ; 0.8),(0.72,0.78,0.92,0.97 ; 1.0)]$ $[(0.0875,0.1200,0.1600,0.1825 ; 0.8),(0.04,0.10,0.18,0.23 ; 1.0)]$ $[(0.4025,0.4525,0.5375,0.5675 ; 0.8),(0.32,0.41,0.58,0.65 ; 1.0)]$

$[(0.0075,0.0075,0.0150,0.0525 ; 0.8),(0.0,0.0,0.020,0.070 ; 1.0)]$ $[(0.4025,0.4525,0.5375,0.5675 ; 0.8),(0.32,0.41,0.58,0.65 ; 1.0)]$ $[(0.0075,0.0075,0.0150,0.0525 ; 0.8),(0.0,0.0,0.020,0.070 ; 1.0)]$ $[(0.4025,0.4525,0.5375,0.5675 ; 0.8),(0.32,0.41,0.58,0.65 ; 1.0)]$ $[(1.0000,1.0000,1.0000,1.0000 ; 1.0),(1.000,1.000,1.0,1.0 ; 1.0)])$

Step 2. We first determine fuzzy density of each decision maker, and its $\lambda$ parameter. Suppose that $\mu\left(e_{1}\right)=$
$0.4, \mu\left(e_{2}\right)=0.4, \mu\left(e_{3}\right)=0.4$, then $\lambda$ of expert can be determined:

$\lambda_{1}=-0.44$. According to Eq. (2.2), we have $\mu\left(e_{1}\right.$, $\left.e_{2}\right)=\mu\left(e_{1}, e_{3}\right)=\mu\left(e_{2}, e_{3}\right)=0.73, \mu\left(e_{1}, e_{2}, e_{3}\right)$ $=1$.

Step 3. By Definition 3.1, $\tilde{a}_{i j}^{k}$ is reordered such that $\tilde{a}_{i j}^{(k)} \leq$ $\tilde{a}_{i j}^{(k+1)}$, then utilize the GITFNGA operator

$$
\begin{aligned}
& \tilde{a}_{i j}=\operatorname{GITFNGA}_{\mu}\left(\tilde{a}_{i j}^{1}, \tilde{a}_{i j}^{2}, \tilde{a}_{i j}^{3}\right) \\
& =\left[\left(\prod_{k=1}^{3}\left(a_{1 i j(k)}^{L}\right)^{\mu\left(A_{(k)}\right)-\mu\left(A_{(k+1)}\right)},\right.\right. \\
& \prod_{k=1}^{3}\left(a_{2 i j(k)}^{L}\right)^{\mu\left(A_{(k)}\right)-\mu\left(A_{(k+1)}\right)}, \\
& \prod_{k=1}^{3}\left(a_{3 i j(k)}^{L}\right)^{\mu\left(A_{(k)}\right)-\mu\left(A_{(k+1)}\right)}, \\
& \left.\prod_{i=1}^{3}\left(a_{4 i j(k)}^{L}\right)^{\mu\left(A_{(k)}\right)-\mu\left(A_{(k+1)}\right)} ; \min _{i=1}^{3} h_{a_{i j}(k)}^{L}\right), \\
& \left(\prod_{i=1}^{3}\left(a_{1 i j(k)}^{L}\right)^{\mu\left(A_{(k)}\right)-\mu\left(A_{(k+1)}\right)},\right. \\
& \prod_{i=1}^{3}\left(a_{2 i j}^{L}(k)\right)^{\mu\left(A_{(k)}\right)-\mu\left(A_{(k+1)}\right)}, \\
& \prod_{i=1}^{3}\left(a_{3 i j}^{L}(k)\right)^{\mu\left(A_{(k)}\right)-\mu\left(A_{(k+1)}\right)}, \\
& \left.\left.\prod_{i=1}^{3}\left(a_{4 i j(k)}^{L}\right)^{\mu\left(A_{(k)}\right)-\mu\left(A_{(k+1)}\right)} ; \min _{i=1}^{3} h_{a_{i j}(k)}^{U}\right)\right]
\end{aligned}
$$

to aggregate all the generalized interval-valued trapezoidal fuzzy decision matrices $R^{k}=\left(\tilde{a}_{i j}\right)_{m \times n}$ $(k=1,2,3)$ into a complex generalized interval trapezoidal fuzzy decision matrix $R=\left(\tilde{a}_{i j}\right)_{m \times n}$ as follows: 
$R=\left(\begin{array}{l}{[(0.3478,0.4001,0.4731,0.5041 ; 0.8),(0.2525,0.3623,0.5086,0.5763 ; 1.0)]} \\ {[(0.0830,0.0965,0.1371,0.2737 ; 0.8),(0.0000,0.0000,0.2957,0.2528 ; 1.0)]} \\ {[(0.3478,0.4001,0.4731,0.5041 ; 0.8),(0.2525,0.3623,0.5086,0.5763 ; 1.0)]} \\ {[(0.9194,0.9416,0.9652,0.9717 ; 0.8),(0.8935,0.9289,0.9777,0.9918 ; 1.0)]} \\ {[(0.7213,0.7546,0.8123,0.8311 ; 0.8),(0.6596,0.7242,0.8398,0.8813 ; 1.0)]}\end{array}\right.$

$[(0.8998,0.9359,0.9623,0.9688 ; 0.8),(0.8679,0.9214,0.9777,0.9918 ; 1.0)]$ $[(0.1611,0.2041,0.2598,0.2873 ; 0.8),(0.0919,0.1758,0.2874,0.3485 ; 1.0)]$ $[(0.3754,0.4316,0.4953,0.5224 ; 0.8),(0.2797,0.3969,0.5258,0.5833 ; 1.0)]$ $[(0.3478,0.4001,0.4731,0.5041 ; 0.8),(0.2525,0.3623,0.5086,0.5763 ; 1.0)]$ $[(0.1611,0.2041,0.2598,0.2873 ; 0.8),(0.0919,0.1758,0.2874,0.3485,1.0)]$

$[(0.0830,0.0965,0.1371,0.2737 ; 0.8),(0.0000,0.0000,0.2957,0.2528 ; 1.0)]$ $[(0.7059,0.7500,0.8098,0.8286 ; 0.8),(0.6408,0.7183,0.8398,0.8813 ; 1.0)]$ $[(0.8447,0.8792,0.9265,0.9406 ; 0.8),(0.7976,0.8546,0.9512,0.9819,1.0)]$ $[(0.0875,0.1200,0.1600,0.1825 ; 0.8),(0.0400,0.1000,0.1800,0.2300 ; 1.0)]$ $[(0.0830,0.0965,0.1371,0.2052 ; 0.8),(0.0000,0.0000,0.1588,0.2528 ; 1.0)]$

$[(0.0830,0.0965,0.1371,0.2737 ; 0.8),(0.0000,0.0000,0.2957,0.2528 ; 1.0)])$ $[(0.7059,0.7500,0.8098,0.8286,0.8),(0.6408,0.7183,0.8398,0.8813 ; 1.0)]$ $[(0.0200,0.0227,0.0387,0.1639 ; 0.8),(0.0000,0.0000,0.1917,0.1127 ; 1.0)]$ $[(0.5251,0.5726,0.6562,0.6847 ; 0.8),(0.4426,0.5303,0.6975,0.7629 ; 1.0)]$ $[(0.9194,0.9416,0.9652,0.9717 ; 0.8),(0.8935,0.9289,0.9777,0.9918 ; 1.0)])$

Step 4. Since $[(1,1,1,1 ; 1),(1,1,1,1 ; 1)]$ and $[(0,0$, $0,0 ; 0),(0,0,0,0 ; 0)]$ are the largest and smallest generalized interval trapezoidal fuzzy numbers, respectively. For cost criteria $c_{1}, c_{4}$ and benefit criteria $c_{2}, c_{3}$ GIT-FPIS $\tilde{\alpha}^{+}$and GIT-FNIS $\tilde{\alpha}^{-}$can be simply denoted as follows:

$\tilde{\alpha}^{+}=([(0,0,0,0 ; 0),(0,0,0,0 ; 0)] \quad[(1,1,1,1 ; 1),(1,1,1,1 ; 1)]$ $[(1,1,1,1 ; 1),(1,1,1,1 ; 1)][(0,0,0,0 ; 0),(0,0,0,0 ; 0)])$

$\tilde{\alpha}^{-}=([(1,1,1,1 ; 1),(1,1,1,1 ; 1)][(0,0,0,0 ; 0),(0,0,0,0 ; 0)]$ $[(0,0,0,0 ; 0),(0,0,0,0 ; 0)][(1,1,1,1 ; 1),(1,1,1,1 ; 1)])$

Denote the alternatives $a_{i}(i=1,2, \ldots, 5)$ by $x_{i}=$ $\left(\begin{array}{llll}\tilde{a}_{i 1} & \tilde{a}_{i 2} & \tilde{a}_{i 3} & \tilde{a}_{i 4}\end{array}\right)$ :

Step 5. We determine fuzzy density of each criterion, and its parameter. Suppose that $\mu\left(c_{1}\right)=0.4, \mu\left(c_{2}\right)=0.25$, $\mu\left(c_{3}\right)=0.37, \mu\left(c_{4}\right)=0.20$, according to Eq. (2.3), the $\lambda$ of criteria can be determined: $\lambda_{2}=-0.44$. By Eq. (2.2), we have $\mu\left(c_{1}, c_{2}\right)=0.6, \mu\left(c_{1}, c_{3}\right)=0.7$, $\mu\left(c_{1}, c_{4}\right)=0.56, \mu\left(c_{2}, c_{3}\right)=0.68, \mu\left(c_{2}, c_{4}\right)=$ $0.43, \mu\left(c_{3}, c_{4}\right)=0.54, \mu\left(c_{1}, c_{2}, c_{3}\right)=0.88, \mu\left(c_{1}\right.$, $\left.c_{2}, c_{4}\right)=0.75, \mu\left(c_{2}, c_{3}, c_{4}\right)=0.73, \mu\left(c_{1}, c_{3}, c_{4}\right)$ $=0.84, \mu\left(c_{1}, c_{2}, c_{3}, c_{4}\right)=1$.

Step 6. According to Eqs. (5.1) and (5.2), respectively, we calculate that

$d_{1}\left(x_{1}, \tilde{\alpha}^{+}\right)=0.344029, d_{1}\left(x_{1}, \tilde{\alpha}^{-}\right)=0.7281649$, $d_{2}\left(x_{2}, \tilde{\alpha}^{+}\right)=0.589855, d_{2}\left(x_{2}, \tilde{\alpha}^{-}\right)=0.4898953$, $d_{3}\left(x_{3}, \tilde{\alpha}^{+}\right)=0.317579, d_{3}\left(x_{3}, \tilde{\alpha}^{-}\right)=0.7377927$,

$$
d_{4}\left(x_{4}, \tilde{\alpha}^{+}\right)=0.704097, d_{4}\left(x_{4}, \tilde{\alpha}^{-}\right)=0.335438
$$$$
d_{5}\left(x_{5}, \tilde{\alpha}^{+}\right)=0.823684, d_{5}\left(x_{5}, \tilde{\alpha}^{-}\right)=0.2014597 .
$$

Step 7. According to Eq. (5.3), we calculate the closeness coefficient of each alternative as follows:

$r\left(x_{1}\right)=0.6791, r\left(x_{2}\right)=0.4537, r\left(x_{3}\right)=0.699$, $r\left(x_{4}\right)=0.32268, r\left(x_{5}\right)=0.1965$.

Step 8. Rank all the alternatives $a_{i}(i=1,2, \ldots, 5)$ according to the closeness coefficient $r\left(x_{i}\right)$ : $a_{3} \succ a_{1} \succ a_{2} \succ a_{4} \succ a_{5}$.

Thus the most desirable alternative is $a_{3}$.

\section{Conclusion}

We have studied the situation that the attributes in the decision making problem are interactive or inter-dependent and the evaluation values are GITFNs. We have defined an aggregation operator with Choquet integral for generalized interval-valued trapezoidal fuzzy group decision making process based TOPSIS, where the inter-dependent of attributes is considered. The GITFS is the best way to deal with uncertainty. GIT FNGA operator is used to aggregate the values of decision makers. Generalized interval-valued trapezoidal fuzzy positive and negative ideal solution calculated by using distance based on Choquet integral. The relative closeness coefficient is used to rank alternatives. The properties of GITFNGA operator are studied, such as idem- 
potency, commutativity, boundedness and monotonicity. We have applied this operator to the multi-criteria generalized interval-valued trapezoidal fuzzy group decision making with Choquet integral-based TOPSIS. Finally, an example has been provided to show the feasibility of our proposed decision making method. The proposed method is different from all the previous techniques for group decision making due to the fact that the proposed method uses generalized interval trapezoidal fuzzy set theory with interdependent criteria taken into account and GITFNGA operator. So it is efficient and feasible for real-world decision making applications. In future, we plan to continue working in the extension and application of the developed multi-criteria generalized interval trapezoidal fuzzy group decision making with Choquet integral-based TOPSIS to some extended forms of intuitionistic spaces and other domains.

Acknowledgments Authors are thankful to the editor and anonymous reviewers, whose insightful comments and constructive suggestions helped us to improve the presentation of the paper.

Open Access This article is distributed under the terms of the Creative Commons Attribution 4.0 International License (http://creativecomm ons.org/licenses/by/4.0/), which permits unrestricted use, distribution, and reproduction in any medium, provided you give appropriate credit to the original author(s) and the source, provide a link to the Creative Commons license, and indicate if changes were made.

\section{References}

1. Angilella, S., Corrente, S., Greco, S., Slowinski, R.: Multiple Criteria Hierarchy Process for the Choquet Integral. Evolut. MultiCriterion Optim. Lect. Notes Comput. Sci. 7811, 475-489 (2013)

2. Ashtiani, B., Haghighirad, F., Makui, A., Montazer, G.: Extension of fuzzy TOPSIS method based on interval-valued fuzzy sets. Appl. Soft Comput. 9(2), 457-461 (2009)

3. Atanassov, K.: Operators over interval-valued intuitionistic fuzzy sets. Fuzzy Sets Syst. 64(2), 159-174 (1994)

4. Atanassov, K., Gargov, G.: Interval-valued intuitionistic fuzzy sets. Fuzzy Sets Syst. 31, 343-349 (1989)

5. Baležentis, T., Zeng, S.: Group multi-criteria decision making based upon interval-valued fuzzy numbers: an extension of the MULTIMOORA method. Expert Syst. Appl. 40, 543-550 (2013)

6. Beg, I., Rashid, T.: TOPSIS for hesitant fuzzy linguistic term sets. Int. J. Intell. Syst. 28, 1162-1171 (2013)

7. Beg, I., Rashid, T.: Multi-criteria trapezoidal valued intuitionistic fuzzy decision making with Choquet integral based TOPSIS. OPSEARCH 51(1), 98-129 (2014)

8. Behzadian, M., Otaghsara, S.K., Yazdani, M., Ignatius, J.: A stateof the-art survey of TOPSIS applications. Expert Syst. Appl. 39, 13051-13069 (2012)

9. Büyüközkan, G., Ruan, D.: Choquet integral based aggregation approach to software development risk assessment. Inf. Sci. 180, 441-451 (2010)

10. Chen, C.T.: Extensions of the TOPSIS for group decision-making under fuzzy environment. Fuzzy Sets Syst. 114, 1-9 (2000)

11. Chen, T.Y.: Signed distance-based TOPSIS method for multiple criteria decision analysis based on generalized interval-valued fuzzy numbers. Int. J. Inf. Technol. Decis. Mak. 6, 1131-1159 (2011)
12. Chen, S.J., Hwang, C.L.: Fuzzy Multiple Attribute Decision Making. Springer, Berlin (1992)

13. Chen, S.M., Lee, L.W.: Fuzzy multiple attributes group decisionmaking based on the interval type-2 TOPSIS method. Expert Syst. Appl. 37(4), 2790-2798 (2010)

14. Chen, T.Y., Tsao, C.Y.: The interval-valued fuzzy TOPSIS method and experimental analysis. Fuzzy Sets Syst. 159, 1410-1428 (2008)

15. Choquet, G.: Theory of capacities. Annales del Institut Fourier 5, 131-295 (1953)

16. Chu, T.C., Lin, Y.C.: An interval arithmetic based fuzzy TOPSIS model. Expert Syst. Appl. 36, 10870-10876 (2009)

17. Demirel, T., Demirel, N.C., Kahraman, C.: Multi-criteria warehouse location selection using Choquet integral. Expert Syst. Appl. 37, 3943-3952 (2010)

18. Dubois, D.: The role of fuzzy sets in decision sciences: old techniques and new directions. Fuzzy Sets Syst. 184, 3-28 (2011)

19. Grabisch, M., Murofushi, T., Sugeno, M.: Fuzzy Measure and Integrals. Physica-Verlag, New York (2000)

20. Herrera, F., Herrera-Viedma, E., Martinez, L.: A fusion approach for managing multi-granularity linguistic term sets in decision making. Fuzzy Sets Syst. 114, 43-58 (2000)

21. Hwang, C.L., Yoon, K.: Multiple Attributes Decision Making Methods and Applications. Springer, Berlin, Heidelberg (1981)

22. Ishii, K., Sugeno, M.: A model human evaluation process using fuzzy measure. Int. J. Man-Mach. Stud. 22, 19-38 (1985)

23. Jahanshahloo, G.R., Lotfi, H.F., Izadikhah, M.: Extension of the TOPSIS method for decision-making problems with fuzzy data. Appl. Math. Comput. 181(2), 1544-1551 (2006)

24. Keeney, R.L., Raiffa, H.: Decision with Multiple Objectives. Wiley, New York (1976)

25. Klement, E.P., Mesiar, R., Pap, E.: Triangular Norms. Kluwer Academic Publishers, Dordrecht (2000)

26. Kojadinovic, I.: Modeling interaction phenomena using fuzzy measures: on the notions of interaction and independence. Fuzzy Sets Syst. 135, 317-340 (2002)

27. Li, D.F.: TOPSIS-based nonlinear-programming methodology for multiattribute decision making with interval-valued intuitionistic fuzzy sets. IEEE Trans. Fuzzy Syst. 18(2), 299-311 (2010)

28. Li, X., Chen, X.: Multi-criteria group decision making based on trapezoidal intuitionistic fuzzy information. Appl. Soft Comput. 30, 454-461 (2015)

29. Liginlal, D., Ow, T.T.: Modeling attitude to risk in human decision processes: an application of fuzzy measures. Fuzzy Sets Syst. 157, 3040-3054 (2006)

30. Mahdavi, I., Mahdavi-Amiri, N., Heidarzade, A., Nourifar, R.: Designing a model of fuzzy TOPSIS in multiple criteria decision making. Appl. Math. Comput. 206, 607-617 (2008)

31. Meyer, P., Roubens, M.: On the use of the Choquet integral with fuzzy numbers in multiple criteria decision support. Fuzzy Sets Syst. 157, 927-938 (2006)

32. Negi, D.S.: Fuzzy analysis and optimization. Ph.D. thesis, Department of Industrial Engineering, Kansas State University (1989)

33. Onisawa, T., Sugeno, M., Nishiwaki, Y., Kawai, H., Harima, Y.: Fuzzy measure analysis of public attitude towards the use of nuclear energy. Fuzzy Sets Syst. 120, 259-289 (1986)

34. Rashid, T., Beg, I., Husnine, S.M.: Robot selection by using generalized interval-valued fuzzy numbers with TOPSIS. Appl. Soft Comput. 21, 462-468 (2014)

35. Sambuc, R.: Fonctions $\Phi$-floues. Application a l'aide au diagnostic en pathologie thyroidienne. Ph.D. thesis, University of Marseille, France (1975)

36. Su, Z.X., Xia, G.P., Chen, M.Y., Wang, L.: Induced generalized intuitionistic fuzzy OWA operator for multi-attribute group decision making. Expert Syst. Appl. 39, 1902-1920 (2012)

37. Sugeno, M.: Theory of fuzzy integral and its application. Doctorial Dissertation, Tokyo Institute of Technology (1974) 
38. Tan, C.: A multi-criteria interval-valued intuitionistic fuzzy group decision making with Choquet integral-based TOPSIS. Expert Syst. Appl. 38, 3023-3033 (2011)

39. Tan, C.Q., Chen, X.H.: Intuitionistic fuzzy Choquet integral operator for multi-criteira decision making. Expert Syst. Appl. 37, 149-157 (2010)

40. Wakker, P.: Additive Representations of Preferences. Kluwer Academic Publishers, Dordrecht (1989)

41. Wei, G.W.: Some geometric aggregation functions and their application to dynamic multiple attribute decision making in the intuitionistic fuzzy setting. Int. J. Uncertainty Fuzziness Knowl.Based Syst. 17(2), 179-196 (2009)

42. Xu, Z.S., Chen, J.: An interactive method for fuzzy multiple attribute group decision making. Inf. Sci. 177(1), 248-263(2007)
43. Yang, W., Chen, Z.: The quasi-arithmetic intuitionistic fuzzy OWA operators. Knowl.-Based Syst. 27, 219-233 (2012)

44. Yao, Y.S., Lin, F.T.: Constructing a fuzzy flow-shop sequencing model based on statistical data. Int. J. Approx. Reason. 29, 215234 (2002)

45. Zadeh, L.A.: Fuzzy sets. Inf. Control 8, 338-356 (1965) 